\title{
A COMBINED SDLINAR(p) MODEL AND IDENTIFICATION AND PREDICTION OF ITS LATENT COMPONENTS
}

\author{
Miodrag S. Djordjević
}

\begin{abstract}
The main subject of this paper is a combined integer-valued autoregressive time series with both positive and negative values, based on a new thinning operator. Some important properties are analyzed. Estimators of the unknown parameters are derived and their asymptotic behavior is analyzed. A simulation and an application on real-data are also shown. In the end, a mechanism for identification and prediction of the latent dimensions of the model are presented.
\end{abstract}

Keywords: Autoregressive time series; Thinning operator; Marginal distribution; Marginal.

\section{Introduction}

Since the last quarter of the previous century there has been a permanent enlargement of application field of integer-valued time series. There is a particular interest in time series with small integer values, since they cannot be approximated by continuous time series. Models that showed a great ability to fit a numerous group of phenomena and processes, in both nature and society are models from the class of integer-valued auto-regressive $(I N A R)$ time series, based on the thinning operators. They were introduced by McKenzie in [14] and Al-Osh and Alzaid [2]. In these two papers, the binomial thinning operator with the counting series of Bernoulli distributed random variables was used. To the present day, there is a variety of INAR models with different marginals. McKenzie[15], Du and Li[9] and AlOsh and Alzaid[1] analyzed models with negative binomial and geometric marginals. Freeland and McCabe[11] studied model with Poisson marginals, Ristić et al.[18] introduced a process with geometric marginals based on a negative binomial thinning operator. Later, integer-valued processes with both positive and negative values appeared. Freeland[10] introduced a process with symmetric Skellam marginal distribution. Chesneau and Kachour[7] defined signed INAR process. Then, Nastić et

Received March 03, 2015; Accepted July 24, 2016

2010 Mathematics Subject Classification. Primary 62M10; Secondary 62M05, 62M20 
al.[17] studied a processes with symmetric discrete Laplace marginals and BarretoSouza and Bourguignon[3] processes with skew discrete Laplace marginals. Djordjević[8] gave a generalization of series with discrete Laplace marginal distribution defining $S D L I N A R(1)$, a model with all four different parameters.

Side by side with a complexity of real-life problems demanding mathematical models, new INAR processes were developed. Systems where current states do not depend only on the previous but on the last $p$ states asked for $I N A R$ models of higher order. Kim and Park[13] defined a integer-valued autoregressive process of order $p$ with signed binomial thinning, $\operatorname{INARS}(p)$. Kachour and Truquet[12] also considered the $I N A R$ process of order $p$ with signed binomial thinning. Zhang et al.[25] introduced a pth-order integer-valued autoregressive process with signed generalized power series thinning operator, GINARS(p). Ristić and Nastić[19] focused on mixtures of geometrically distributed counting series. Also, Zhu and Joe[26] and Weiss[22] developed a combined INAR model of $p$ th-order, applicable in situations where the current state depends, with some probability, on one of the last $p$ states. Model presented in this paper is one of that kind. It relies on the results given in [16].

In Section 2, the definition of the thinning operator $(\alpha, \beta) \odot$ and some of its important properties are shown. In Section 3 we introduce a new model of order $p$, we give its definition and characterization through important properties. Then, in Section 4, conditional least square and Yule-Walker estimators are derived and their asymptotic behavior is described. Finally, in Section 5, we demonstrate identification and prediction of latent components using realization of the defined process. Also, the results of simulation and application on real data are shown.

\section{The thinning operator $(\alpha, \beta) \odot$}

First, we will give the definition of the thinning operator $(\alpha, \beta) \odot$, introduced in [8], which represents a generalization of thinning operators presented in [17] and [3]. Let the random variable $Z$ has skew discrete Laplace distribution, $S D L(\mu /(1+$ $\mu), \nu(1+\nu))$, with parameters $\mu /(1+\mu), \mu>0$ and $\nu /(1+\nu), \nu>0$. The thinning operator $(\alpha, \beta) \odot$ is defined in the following way

$$
((\alpha, \beta) \odot Z)|Z \stackrel{d}{=}(\alpha * X-\beta * Y)|(X-Y),
$$

where $\alpha *$ and $\beta *$ are the negative binomial thinning operators, defined in [18] as $\alpha * X=\sum_{i=1}^{X} W_{i}$, where $\left\{W_{i}\right\}$ is a sequence of independent and identically distributed random variables with geometric, $\operatorname{Geom}(\alpha /(1+\alpha)), \alpha \in(0,1)$, distribution, where $W_{i}$ and $X$ are independent random variables for all $i \geqslant 1$ and $\beta * Y=\sum_{i=1}^{Y} V_{i}$, where $\left\{V_{i}\right\}$ is a sequence of independent and identically distributed random variables with geometric, $\operatorname{Geom}(\beta /(1+\beta)), \beta \in(0,1)$, and $V_{i}$ and $Y$ are independent random variables for all $i \geqslant 1$. Random variables $X$ and $Y$ are independent and have geometric, $\operatorname{Geom}(\mu /(1+\mu))$ and $\operatorname{Geom}(\nu /(1+\nu))$, distributions, respectively. Also, the counting series in $\alpha * X$ and $\beta * Y,\left\{W_{i}\right\}$ and $\left\{V_{i}\right\}$ are mutually independent 
with geometric, $\operatorname{Geom}(\alpha /(1+\alpha))$ and $\operatorname{Geom}(\beta /(1+\beta))$, distributions, respectively. It should be said that parameters $\mu, \nu, \alpha$ and $\beta$ represent the mean value of the corresponding geometric distributions. Several important properties of the random variable $(\alpha, \beta) \odot Z$ will be mentioned.

Proposition 2.1. The random variable $(\alpha, \beta) \odot Z$ has the following properties:

1. The characteristic function of the random variable $(\alpha, \beta) \odot Z$ is

$$
\varphi_{(\alpha, \beta) \odot Z}(t)=\frac{\left(1+\alpha-\alpha e^{i t}\right)\left(1+\beta-\beta e^{-i t}\right)}{\left(1+\alpha(1+\mu)-\alpha(1+\mu) e^{i t}\right)\left(1+\beta(1+\nu)-\beta(1+\nu) e^{-i t}\right)} ;
$$

2. $E((\alpha, \beta) \odot Z)=\alpha \mu-\beta \nu$;

3. $\operatorname{Var}((\alpha, \beta) \odot Z)=\alpha \mu(1+2 \alpha+\alpha \mu)+\beta \nu(1+2 \beta+\beta \nu)$;

4. $E((\alpha, \beta) \odot Z \mid Z)=(\alpha-\beta) \frac{\mu \nu}{1+\mu+\nu}+\alpha Z I_{\{Z \geq 0\}}+\beta Z I_{\{Z<0\}}$;

5. $\operatorname{Var}((\alpha, \beta) \odot Z \mid Z)=(\alpha-\beta)^{2} \frac{\mu(1+\mu) \nu(1+\nu)}{(1+\mu+\nu)^{2}}+\mu \nu \frac{\alpha(1+\alpha)+\beta(1+\beta)}{1+\mu+\nu}$

$$
+\alpha(1+\alpha) Z I_{\{Z \geq 0\}}-\beta(1+\beta) Z I_{\{Z<0\}} \text {. }
$$

Using the thinning operator $(\alpha, \beta) \odot, S D L I N A R(1)$ time series model is defined as $Z_{n} \stackrel{d}{=}(\alpha, \beta) \odot Z_{n-1}+e_{n}, n \geq 1$, where random variables $Z_{n}, n \geq 0$ have a skew discrete Laplace distribution, $S D L(\mu /(1+\mu), \nu /(1+\nu))$. The $S D L I N A R(1)$ time series is based on the NGINAR(1) model, introduced by [18]. Since in the $N G I N A R(1)$ model has the restriction that $\alpha \in(0, \mu /(1+\mu)]$, the $\operatorname{SDLINAR(1)}$ model must have it too. It means that to have well-defined distribution of $\left\{e_{n}\right\}$, as discussed in [18] and [8], it is necessary to have $\alpha \in(0, \mu /(1+\mu)]$ and $\beta \in(0, \nu /(1+$ $\nu)$. Random variables $e_{n}, n \geq 1$, are independent and identically distributed integervalued random variables. Also, $e_{n}$ and $Z_{m}$ are independent for all $m<n$.

\section{Construction and properties of the model}

Using the operator $(\alpha, \beta) \odot$, we define a combined integer-valued autoregressive time series $\left\{Z_{n}, n \geq 0\right\}$ as follows

$$
Z_{n}=\left\{\begin{array}{cc}
(\alpha, \beta) \odot_{n} Z_{n-1}+e_{n}, & \text { w.p. } \phi_{1}, \\
(\alpha, \beta) \odot_{n} Z_{n-2}+e_{n}, & \text { w.p. } \phi_{2}, \\
\vdots & \vdots \\
(\alpha, \beta) \odot_{n} Z_{n-p}+e_{n}, & \text { w.p. } \phi_{p},
\end{array}, n>p\right.
$$

where $0 \leq \phi_{1}, \phi_{2}, \ldots, \phi_{p} \leq 1, \sum_{i=1}^{p} \phi_{i}=1, p \geq 1$, "w.p." stands for "with probability" and the following conditions are satisfied

1. $\left\{e_{n}\right\}$ is a sequence of i.i.d. random variables and each random variable $e_{n}$ is independent of all $Z_{m}$ and $(\alpha, \beta) \odot_{m+i} Z_{m}$, where $m<n$ and $i=1, \ldots, p$, 
2. the counting series of the thinning operators applied at the moment $m$ are independent of each other and independent of the sequence $\left\{e_{n}\right\}$,

3. the counting series of the thinning operator applied on the random variable $Z_{n}$ and the random variables $Z_{n-1}, Z_{n-2}, \ldots$ are independent,

The subscript $n$ in $(\alpha, \beta) \odot_{n}$ denotes that the thinning operator is applied in the time moment $n$, i.e. while the random variable $Z_{n}$ is being generated. Since the thinning operator could be applied up to $p$ times on the same variable $Z_{k}$, it is necessary to label the moment when the operator is applied.

If the time series $\left\{Z_{n}\right\}$ has skew discrete Laplace $S D L(\mu /(1+\mu), \nu /(1+\nu))$, marginal distribution, where $\alpha \in(0, \mu /(1+\mu)]$ and $\beta \in(0, \nu /(1+\nu)]$, we get the model which will be called the Combined Skew Discrete Laplace $I N A R$ process of order $p$ or $C S D L I N A R(p)$.

In the following proposition some of the basic properties of the $C S D L I N A R(p)$ model are shown.

Proposition 3.1. The innovation process $\left\{e_{n}\right\}$ has the following properties:

- The distribution of the random variable $e_{n}$ is

$$
e_{n} \stackrel{d}{=} \begin{cases}S D L\left(\frac{\mu}{1+\mu}, \frac{\nu}{1+\nu}\right), & \text { w.p. }\left(1-\frac{\alpha \mu}{\mu-\alpha}\right)\left(1-\frac{\beta \nu}{\nu-\beta}\right), \\ S D L\left(\frac{\mu}{1+\mu}, \frac{\beta}{1+\beta}\right), & \text { w.p. }\left(1-\frac{\alpha \mu}{\mu-\alpha}\right) \frac{\beta \nu}{\nu-\beta}, \\ S D L\left(\frac{\alpha}{1+\alpha}, \frac{\nu}{1+\nu}\right), & \text { w.p. } \frac{\alpha \mu}{\mu-\alpha}\left(1-\frac{\beta \nu}{\nu-\beta}\right), \\ S D L\left(\frac{\alpha}{1+\alpha}, \frac{\beta}{1+\beta}\right), & \text { w.p. } \frac{\alpha \mu}{\mu-\alpha} \frac{\beta \nu}{\nu-\beta} .\end{cases}
$$

- $E\left(e_{n}\right)=\mu(1-\alpha)-\nu(1-\beta)$ and

- $\operatorname{Var}\left(e_{n}\right)=\mu(1+\alpha)[(1+\mu)(1-\alpha)-\alpha]+\nu(1+\beta)[(1+\nu)(1-\beta)-\beta]$.

Proof. Using the definition (3.1), independence of random variables $(\alpha, \beta) \odot_{m}$ $Z_{n-i}, i=1, \ldots, p$ and $e_{n}$ and the fact that all $Z_{n-i}, i=1, \ldots, p$ have the same distribution, we get the characteristic function of the random variable $e_{n}$ in the following way.

$$
\begin{aligned}
\varphi_{Z_{n}}(t) & =\sum_{i=1}^{p} \phi_{i} \varphi_{(\alpha, \beta) \odot_{n} Z_{n-i}}(t) \varphi_{e_{n}}(t)=\varphi_{e_{n}}(t) \sum_{i=1}^{p} \phi_{i} \varphi_{(\alpha, \beta) \odot_{n} Z_{n-i}}(t) \\
& =\varphi_{e_{n}}(t) \sum_{i=1}^{p} \phi_{i} \varphi_{(\alpha, \beta) \odot_{n} Z}(t)=\varphi_{e_{n}}(t) \varphi_{(\alpha, \beta) \odot_{n} Z}(t)
\end{aligned}
$$

where the random variable $Z$ has $S D L(\mu /(1+\mu), \nu /(1+\nu))$ distribution. Hence, 
we get that

$$
\begin{aligned}
\varphi_{e_{n}}(t) & =\frac{\varphi_{Z_{n}}(t)}{\varphi_{(\alpha, \beta) \odot_{n} Z}(t)}=\frac{\left(1+\alpha(1+\mu)-\alpha(1+\mu) e^{i t}\right)\left(1+\beta(1+\nu)-\beta(1+\nu) e^{-i t}\right)}{\left(1+\mu-\mu e^{i t}\right)\left(1+\nu-\nu e^{-i t}\right)\left(1+\alpha-\alpha e^{i t}\right)\left(1+\beta-\beta e^{-i t}\right)} \\
& =\left(\frac{1-\frac{\alpha \mu}{\mu-\alpha}}{1+\mu-\mu e^{i t}}+\frac{\frac{\alpha \mu}{\mu-\alpha}}{1+\alpha-\alpha e^{i t}}\right)\left(\frac{1-\frac{\beta \nu}{\nu-\beta}}{1+\nu-\nu e^{i t}}+\frac{\frac{\beta \nu}{\nu-\beta}}{1+\beta-\beta e^{i t}}\right)
\end{aligned}
$$

After some simple algebraic transformations, $\varphi_{e_{n}}(t)$ becomes

$$
\begin{aligned}
\varphi_{e_{n}}(t) & =\frac{\left(1-\frac{\alpha \mu}{\mu-\alpha}\right)\left(1-\frac{\beta \nu}{\nu-\beta}\right)}{\left(1+\mu-\mu e^{i t}\right)\left(1+\nu-\nu e^{-i t}\right)}+\frac{\left(1-\frac{\alpha \mu}{\mu-\alpha}\right) \frac{\beta \nu}{\nu-\beta}}{\left(1+\mu-\mu e^{i t}\right)\left(1+\beta-\beta e^{-i t}\right)} \\
& +\frac{\frac{\alpha \mu}{\mu-\alpha}\left(1-\frac{\beta \nu}{\nu-\beta}\right)}{\left(1+\alpha-\alpha e^{i t}\right)\left(1+\nu-\nu e^{-i t}\right)}+\frac{\frac{\alpha \mu}{\mu-\alpha} \frac{\beta \nu}{\nu-\beta}}{\left(1+\alpha-\alpha e^{i t}\right)\left(1+\beta-\beta e^{-i t}\right)} \\
& =\left(1-\frac{\alpha \mu}{\mu-\alpha}\right)\left(1-\frac{\beta \nu}{\nu-\beta}\right) \varphi_{1}(t)+\left(1-\frac{\alpha \mu}{\mu-\alpha}\right) \frac{\beta \nu}{\nu-\beta} \varphi_{2}(t) \\
& +\frac{\alpha \mu}{\mu-\alpha}\left(1-\frac{\beta \nu}{\nu-\beta}\right) \varphi_{3}(t)+\frac{\alpha \mu}{\mu-\alpha} \frac{\beta \nu}{\nu-\beta} \varphi_{4}(t),
\end{aligned}
$$

where

$$
\begin{aligned}
& \varphi_{1}(t)=\frac{1}{\left(1+\mu-\mu e^{i t}\right)\left(1+\nu-\nu e^{-i t}\right)}, \quad \varphi_{2}(t)=\frac{1}{\left(1+\mu-\mu e^{i t}\right)\left(1+\beta-\beta e^{-i t}\right)}, \\
& \varphi_{3}(t)=\frac{1}{\left(1+\alpha-\alpha e^{i t}\right)\left(1+\nu-\nu e^{-i t}\right)}, \quad \varphi_{4}(t)=\frac{1}{\left(1+\alpha-\alpha e^{i t}\right)\left(1+\beta-\beta e^{-i t}\right)} .
\end{aligned}
$$

Since these functions are characteristic functions of random variables with the skew discrete Laplace distribution, $S D L\left(\frac{\mu}{1+\mu}, \frac{\nu}{1+\nu}\right), S D L\left(\frac{\mu}{1+\mu}, \frac{\beta}{1+\beta}\right), S D L\left(\frac{\alpha}{1+\alpha}, \frac{\nu}{1+\nu}\right)$ and $S D L\left(\frac{\alpha}{1+\alpha}, \frac{\beta}{1+\beta}\right)$, respectively, the characteristic function of the random variable $e_{n}$ is presented as a probability weighted sum of four characteristic functions. It follows that the random variable $e_{n}$ could be expressed as a mixture of four mentioned variables.

Remark 3.1. In order for the numbers $\left(1-\frac{\alpha \mu}{\mu-\alpha}\right)\left(1-\frac{\beta \nu}{\nu-\beta}\right),\left(1-\frac{\alpha \mu}{\mu-\alpha}\right) \frac{\beta \nu}{\nu-\beta}, \frac{\alpha \mu}{\mu-\alpha} \frac{\beta \nu}{\nu-\beta}$ and $\frac{\alpha \mu}{\mu-\alpha}\left(1-\frac{\beta \nu}{\nu-\beta}\right)$ to be probabilities, it is necessary that $\alpha \in(0, \mu /(1+\mu)]$ and $\beta \in$ $(0, \nu /(1+\nu)]$.

Using the obtained characteristic function, i.e. its first and second derivation, we get the mean and the variation of the innovation process.

Regarding the form of the distribution of the random variable $e_{n}$ and the fact that difference of two random variables with geometric distribution is a random variable with discrete Laplace distribution, we can conclude the following. 
Corollary 3.1. If $\alpha \in(0, \mu /(1+\mu)]$ and $\beta \in(0, \nu /(1+\nu)]$, then $e_{n} \stackrel{d}{=} \varepsilon_{n}-\eta_{n}$, where $\varepsilon_{n}$ and $\eta_{n}$ are two independent random variables distributed as

$\varepsilon_{n} \stackrel{d}{=}\left\{\begin{array}{ll}\operatorname{Geom}\left(\frac{\mu}{1+\mu}\right), & \text { w.p. } 1-\frac{\alpha \mu}{\mu-\alpha}, \\ \operatorname{Geom}\left(\frac{\alpha}{1+\alpha}\right), & \text { w.p. } \frac{\alpha \mu}{\mu-\alpha}\end{array}, \eta_{n} \stackrel{d=}{\operatorname{Geom}\left(\frac{\nu}{1+\nu}\right),} \begin{array}{ll}\text { w.p. } 1-\frac{\beta \nu}{\nu-\beta}, \\ \operatorname{Geom}\left(\frac{\beta}{1+\beta}\right), & \text { w.p. } \frac{\beta \nu}{\nu-\beta} .\end{array}\right.$

Using representations of the random variable $(\alpha, \beta) \odot_{n} Z_{n}$ given in the Proposition 2.1(2) and representation of the innovation process $\left\{e_{n}\right\}$ from previous corollary, we can present in distribution the $C S D L I N A R(p)$ time series, $\left\{Z_{n}\right\}$, as a difference of two independent $C G I N A R(p)$ time series, $\left\{X_{n}\right\}$ and $\left\{Y_{n}\right\}$, defined in [16] with geometrical Geom $(\mu /(1+\mu))$ and Geom $(\nu /(1+\nu))$ marginal distributions and with previously mentioned $\left\{\varepsilon_{n}\right\}$ and $\left\{\eta_{n}\right\}$ as their innovation processes.

Remark 3.2. Let $\left\{Z_{n}\right\}$ be a $C S D L I N A R(p)$ time series defined in expression (3.1) and $\left\{X_{n}\right\}$ and $\left\{Y_{n}\right\}$ two independent $C G I N A R(p)$ time series defined as

$$
X_{n}=\left\{\begin{array}{cc}
\alpha *_{n} X_{n-1}+\varepsilon_{n}, & \text { w.p. } \phi_{1}, \\
\alpha *_{n} X_{n-2}+\varepsilon_{n}, & \text { w.p. } \phi_{2}, \\
\vdots & \vdots \\
\alpha *_{n} X_{n-p}+\varepsilon_{n}, & \text { w.p. } \phi_{p},
\end{array} Y_{n}=\left\{\begin{array}{cc}
\beta *_{n} Y_{n-1}+\eta_{n}, & \text { w.p. } \phi_{1}, \\
\beta *_{n} Y_{n-2}+\eta_{n}, & \text { w.p. } \phi_{2}, \\
\vdots & \vdots \\
\beta *_{n} Y_{n-p}+\eta_{n}, & \text { w.p. } \phi_{p},
\end{array}\right.\right.
$$

where $\left\{\varepsilon_{n}\right\}$ and $\left\{\eta_{n}\right\}$ are two mutually independent sequences of identically distributed random variables. Then $Z_{n} \stackrel{d}{=} X_{n}-Y_{n}$.

Remark 3.3. The CSDLINAR(p) process is a Markov process of order $p$. The Markovian property follows from its definition. The transition probabilities of the $C S D L I N A R(p)$ time series $\left\{Z_{n}\right\}$ can be calculated using transition probabilities of $S D L I N A R(1)$ model from $[8]$.

$$
P\left(Z_{n}=z_{n} \mid H_{n-1}\right)=\sum_{i=1}^{p} \phi_{i} P\left(Z_{n}=z_{n} \mid Z_{n-i}=z_{n-i}\right)=\sum_{i=1}^{p} \phi_{i} p_{Z}\left(z_{n}, z_{n-i}\right),
$$

where

$$
p_{Z}(k, j)= \begin{cases}\sum_{m=0}^{\infty} \sum_{l=-\infty}^{\infty} p_{D N B}\left(k-l ; m+j, \frac{\alpha}{1+\alpha}, m, \frac{\beta}{1+\beta}\right) p_{e}(l) & , j \geq 0 \\ \sum_{m=0}^{\infty} \sum_{l=-\infty}^{\infty} p_{D N B}\left(k-l ; m, \frac{\alpha}{1+\alpha}, m-j, \frac{\beta}{1+\beta}\right) p_{e}(l) & , j<0\end{cases}
$$

$p_{D N B}(k ; r, p ; l, q)$ is a probability mass function of the random variable that represents a difference of two independent random variables with a negative binomial distribution,

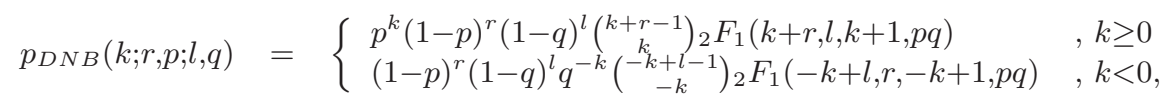


$p_{e}(l)$ is the probability mass function of the innovation process $\left\{e_{n}\right\}$,

$$
p_{e}(l)=P\left\{e_{n}=l\right\}= \begin{cases}\left(\frac{\mu}{1+\mu}\right)^{l}\left(1-\frac{\alpha \mu}{\mu-\alpha}\right) \frac{1+\mu+\beta(1+\nu)}{(1+\mu+\nu)(1+\mu+\beta)} & \\ +\left(\frac{\alpha}{1+\alpha}\right)^{l} \frac{\alpha \mu}{\mu-\alpha} \frac{1+\alpha+\beta(1+\nu)}{(1+\alpha+\nu)(1+\alpha+\beta)}, & l \geq 0 \\ \left(\frac{\nu}{1+\nu}\right)^{-l}\left(1-\frac{\beta \nu}{\nu-\beta}\right) \frac{1+\nu+\alpha(1+\mu)}{(1+\nu+\mu)(1+\nu+\alpha)} & \\ +\left(\frac{\beta}{1+\beta}\right)^{-l} \frac{\beta \nu}{\nu-\beta} \frac{1+\beta+\alpha(1+\mu)}{(1+\beta+\mu)(1+\beta+\alpha)}, & l<0,\end{cases}
$$

and ${ }_{2} F_{1}(k, l, m, p)$ is the Gaussian hypergeometric function.

Proposition 3.2. The CSDLINAR(p) process is strictly stationary and ergodic.

Proof. Using the obtained transition probabilities, the Markovian property and the identical distribution of the time series $\left\{Z_{n}\right\}$, strong stationarity can be proven as follows.

Let $A_{n, k}, n, k \in \mathbb{N}$ be the event $\left\{Z_{n+k}=z_{k}, Z_{n+k-1}=z_{k-1}, \ldots, Z_{n+1}=z_{1}\right\}$. To prove strong stationarity it will be enough to show that $P\left(A_{0, k}\right)=P\left(A_{n, k}\right)$.

$$
\begin{aligned}
P\left(A_{0, k}\right) & =P\left(Z_{k}=z_{k} \mid H_{k-1}\right) P\left(Z_{k-1}=z_{k-1}, \ldots, Z_{1}=z_{1}\right) \\
& =P\left(Z_{k}=z_{k} \mid H_{k-1}\right) P\left(Z_{k-1}=z_{k-1} \mid H_{k-2}\right) P\left(Z_{k-2}=z_{k-2}, \ldots, Z_{1}=z_{1}\right) \\
& =P\left(Z_{1}=z_{1}\right) \prod_{i=0}^{k-2} P\left(Z_{k-i}=z_{k-i} \mid H_{k-i-1}\right) .
\end{aligned}
$$

Since transition probabilities $p_{Z}(k, l)$ do not depend on the position of the elements in the series but only on the distance between the elements, we have that $P\left(Z_{k-i}=z_{k-i} \mid H_{k-i-1}\right)=P\left(Z_{n+k-i}=z_{k-i} \mid H_{n+k-i-1}\right)$. Also, all $Z_{i}, i \geq 1$ have the same distribution, hence $P\left(Z_{1}=z_{1}\right)=P\left(Z_{n+1}=z_{1}\right)$. Substituting the mentioned probabilities and tracing back it follows that

$$
P\left(A_{0, k}\right)=P\left(Z_{n+1}=z_{1}\right) \prod_{i=0}^{k-2} P\left(Z_{n+k-i}=z_{k-i} \mid H_{n+k-i-1}\right)=P\left(A_{n, k}\right) .
$$

Regarding the ergodicity, we can use the definition 6.30 from [5] that the stationary process $\left\{Z_{n}\right\}$ is ergodic if every invariant (in respect to the process $\left\{Z_{n}\right\}$ ) event has probability 0 or 1 . According to the Proposition 6.32, also from [5], every invariant event in respect to a stationary process is, also, a tail event. But, for every $n \geq 1, \sigma$-algebra $\mathcal{F}\left(Z_{n}, Z_{n-1}, \ldots\right)$, which is generated by random variables $Z_{n}, Z_{n-1}, \ldots$, is a subset of $\sigma$-algebra $\mathcal{F}\left(e_{n}, \mathbf{W}^{(n)}, \mathbf{V}^{(n)}, e_{n-1}, \mathbf{W}^{(n-1)}, \mathbf{V}^{(n-1)}, \ldots\right)$, where $\mathbf{W}^{(k)}$ and $\mathbf{V}^{(k)}$ are the counting series $\left\{W_{i}\right\}$ and $\left\{V_{i}\right\}$ that generate random variable $Z_{k}$. Due to the independence of the random series $\left\{e_{n}, \mathbf{W}^{(n)}, \mathbf{V}^{(n)}\right\}$, applying the Kolmogorov's $0-1$ law, every tail event has probability 0 or 1 , which confirms the ergodicity of time series $\left\{Z_{n}\right\}$.

In order to simplify some of the following expressions, let $Z_{n}^{+}=Z_{n} I_{\left\{Z_{n} \geq 0\right\}}$ and $Z_{n}^{-}=-Z_{n} I_{\left\{Z_{n}<0\right\}}$. 
Proposition 3.3. One-step ahead conditional expectation and variance of the

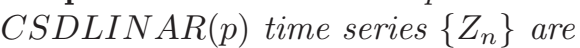

$$
\begin{aligned}
E\left(Z_{n} \mid H_{n-1}\right) & =(\alpha-\beta) \frac{\mu \nu}{1+\mu+\nu}+\mu(1-\alpha)-\nu(1-\beta) \\
& +\alpha \sum_{i=1}^{p} \phi_{i} Z_{n-i}^{+}-\beta \sum_{i=1}^{p} \phi_{i} Z_{n-i}^{-}
\end{aligned}
$$

$$
\begin{aligned}
\operatorname{Var}\left(Z_{n} \mid H_{n-1}\right) & =\operatorname{Var}\left(e_{n}\right)+(\alpha-\beta)^{2} \frac{\mu(1+\mu) \nu(1+\nu)}{(1+\mu+\nu)^{2}} \\
& +\mu \nu \frac{\alpha(1+\alpha)+\beta(1+\beta)}{1+\mu+\nu} \\
& +\alpha \sum_{i=1}^{p} \phi_{i} Z_{n-i}^{+}\left(1+\alpha+\alpha Z_{n-i}^{+}-\alpha \sum_{j=1}^{p} \phi_{j} Z_{n-j}^{+}+\beta \sum_{j=1}^{p} \phi_{j} Z_{n-j}^{-}\right) \\
& +\beta \sum_{i=1}^{p} \phi_{i} Z_{n-i}^{-}\left(1+\beta+\beta Z_{n-i}^{-}+\alpha \sum_{j=1}^{p} \phi_{j} Z_{n-j}^{+}-\beta \sum_{j=1}^{p} \phi_{j} Z_{n-j}^{-}\right) .
\end{aligned}
$$

Proof. Using the property 4. from Proposition 2.1 and Proposition 3.1 it follows that

$$
\begin{aligned}
E\left(Z_{n} \mid H_{n-1}\right) & =\sum_{i=1}^{p} E\left((\alpha, \beta) \odot_{n} Z_{n-i}+e_{n} \mid H_{n-1}\right) \phi_{i} \\
& =\sum_{i=1}^{p} E\left((\alpha, \beta) \odot_{n} Z_{n-i} \mid H_{n-1}\right) \phi_{i}+\sum_{i=1}^{p} E\left(e_{n}\right) \phi_{i} \\
& =(\alpha-\beta) \frac{\mu \nu}{1+\mu+\nu}+\alpha \sum_{i=1}^{p} \phi_{i} Z_{n-i}^{+}-\beta \sum_{i=1}^{p} \phi_{i} Z_{n-i}^{-} \\
& +\mu(1-\alpha)-\nu(1-\beta) . \\
\operatorname{Var}\left(Z_{n} \mid H_{n-1}\right) & =E\left(Z_{n}^{2} \mid H_{n-1}\right)-E\left(Z_{n} \mid H_{n-1}\right)^{2} \\
& =\sum_{i=1}^{p} E\left(\left((\alpha, \beta) \odot_{n} Z_{n-i}+e_{n}\right)^{2} \mid H_{n-1}\right) \phi_{i} \\
& -\left(\sum_{i=1}^{p} E\left(\left((\alpha, \beta) \odot_{n} Z_{n-i}+e_{n}\right) \mid H_{n-1}\right) \phi_{i}\right)^{2} .
\end{aligned}
$$


Again, using the results from Proposition 2.1, the conditional variance becomes

$$
\begin{aligned}
& \operatorname{Var}\left(Z_{n} \mid H_{n-1}\right)=\operatorname{Var}\left(e_{n}\right)+(\alpha-\beta)^{2} \frac{\mu(1+\mu) \nu(1+\nu)}{(1+\mu+\nu)^{2}}+\mu \nu \frac{\alpha(1+\alpha)+\beta(1+\beta)}{1+\mu+\nu} \\
& \quad+\alpha \sum_{i=1}^{p} \phi_{i} Z_{n-i}^{+}\left(1+\alpha+\alpha Z_{n-i}^{+}-\alpha \sum_{j=1}^{p} \phi_{j} Z_{n-j}^{+}+\beta \sum_{j=1}^{p} \phi_{j} Z_{n-j}^{-}\right) \\
& \quad+\beta \sum_{i=1}^{p} \phi_{i} Z_{n-i}^{-}\left(1+\beta+\beta Z_{n-i}^{-}+\alpha \sum_{j=1}^{p} \phi_{j} Z_{n-j}^{+}-\beta \sum_{j=1}^{p} \phi_{j} Z_{n-j}^{-}\right) . \quad \square
\end{aligned}
$$

Regarding the correlation structure, we have the following results

Proposition 3.4. The covariance function of the CSDLINAR(p) time series $\left\{Z_{n}\right\}$ satisfies the following equation:

$$
\gamma_{Z}(k)=\sum_{i=1}^{p}\left[\alpha \gamma_{Z Z^{+}}(k-i)-\beta \gamma_{Z Z^{-}}(k-i)\right] \phi_{i},
$$

where $\gamma_{Z}(k)$ is the autocorrelation function of the time series $\left\{Z_{n}\right\}, \gamma_{Z Z^{+}}(k)$ is the cross-covariance function of lag $k$ between series $\left\{Z_{n}^{+}\right\}$and $\left\{Z_{n}\right\}$ and $\gamma_{Z Z^{-}}(k)$ is the cross-covariance function of lag $k$ between series $\left\{Z_{n}^{-}\right\}$and $\left\{Z_{n}\right\}$, defined in the following way

$$
\begin{aligned}
\gamma_{Z Z^{+}}(k) & \equiv E\left(Z_{n}-E Z_{n}\right)\left(Z_{n+k}^{+}-E Z_{n+k}^{+}\right), \\
\gamma_{Z Z^{-}}(k) & \equiv E\left(Z_{n}-E Z_{n}\right)\left(Z_{n+k}^{-}-E Z_{n+k}^{-}\right) .
\end{aligned}
$$

Proof. Using the Markovian property, we get that

$$
\begin{aligned}
\gamma_{Z}(k)= & \operatorname{Cov}\left(E\left(Z_{n+k} \mid Z_{n}\right), Z_{n}\right)=\operatorname{Cov}\left(E\left(E\left(Z_{n+k} \mid H_{n+k-1}\right) \mid Z_{n}\right), Z_{n}\right) \\
= & \operatorname{Cov}\left(E\left(Z_{n+k} \mid H_{n+k-1}\right), Z_{n}\right) \\
= & \operatorname{Cov}\left((\alpha-\beta) \frac{\mu \nu}{1+\mu+\nu}+\mu(1-\alpha)-\nu(1-\beta)\right. \\
& \left.\quad+\alpha \sum_{i=1}^{p} \phi_{i} Z_{n+k-i}^{+}-\beta \sum_{i=1}^{p} \phi_{i} Z_{n+k-i}^{-}, Z_{n}\right) \\
= & \operatorname{Cov}\left(\alpha \sum_{i=1}^{p} \phi_{i} Z_{n+k-i}^{+}, Z_{n}\right)-\operatorname{Cov}\left(\beta \sum_{i=1}^{p} \phi_{i} Z_{n+k-i}^{-}, Z_{n}\right) \\
= & \sum_{i=1}^{p}\left[\alpha \phi_{i} \operatorname{Cov}\left(Z_{n+k-i}^{+}, Z_{n}\right)-\beta \phi_{i} \operatorname{Cov}\left(Z_{n+k-i}^{-}, Z_{n}\right)\right] \\
= & \sum_{i=1}^{p}\left(\alpha \gamma_{Z Z^{+}}(k-i)-\beta \gamma_{Z Z^{-}}(k-i)\right) \phi_{i} .
\end{aligned}
$$


Proposition 3.5. Since the covariance function $\gamma_{Z}(k)$ could also be presented in the form $\gamma_{Z}(k)=\gamma_{Z Z^{+}}(k)-\gamma_{Z Z^{-}}(k)$, the following recurrent relations stand

$$
\gamma_{Z Z+}(k)=\alpha \sum_{i=1}^{p} \phi_{i} \gamma_{Z Z+}(k-i) \text { and } \gamma_{Z Z^{-}}(k)=\beta \sum_{i=1}^{p} \phi_{i} \gamma_{Z Z-}(k-i) \text {. }
$$

Proof. First, we should prove the equality

$$
E\left(Z_{n+1}^{+} \mid H_{n}\right)=(1-\alpha) \frac{\mu(1+\mu)}{1+\mu+\nu}+\alpha \sum_{i=1}^{p} \phi_{i} Z_{n+1-i}^{+} .
$$

Using the property of the $S D L I N A R(1)$ time series, e.g. $\left\{Z_{(1), n}\right\}$,

$$
E\left(Z_{(1), n+k}^{+} \mid Z_{(1), n}\right)=\left(1-\alpha^{k}\right) \frac{\mu(1+\mu)}{1+\mu+\nu}+\alpha^{k} Z_{(1), n}^{+},
$$

that could easily be proven by mathematical induction, we get the following

$$
\begin{aligned}
E\left(Z_{n+1}^{+} \mid H_{n}\right)= & E\left(Z_{n+1} I_{\left\{Z_{n+1} \geq 0\right\}} \mid H_{n}\right) \\
= & \sum_{i=1}^{p} \phi_{i} E\left(\left((\alpha, \beta) \odot_{n+1} Z_{n+1-i}+e_{n+1}\right)\right. \\
& \left.\times I_{\left\{(\alpha, \beta) \odot_{n+1} Z_{n+1-i}+e_{n+1} \geq 0\right\}} \mid H_{n}\right) \\
= & \sum_{i=1}^{p} \phi_{i} E\left(\left((\alpha, \beta) \odot_{n+1} Z_{n+1-i}+e_{n+1}\right)\right. \\
\left.\quad \times I_{\left\{(\alpha, \beta) \odot_{n+1}\right.} Z_{n+1-i}+e_{n+1} \geq 0\right\} & \left.Z_{n+1-i}\right) \\
= & (1-\alpha) \frac{\mu(1+\mu)}{1+\mu+\nu}+\alpha \sum_{i=1}^{p} \phi_{i} Z_{n+1-i}^{+} .
\end{aligned}
$$

Next, in the similar way as it was done in the proof of the Theorem 8 in [8], it could be derived that

$$
E\left(X_{n+1} \mid H_{n}\right)=(1-\alpha) \frac{\mu(1+\mu)}{1+\mu+\nu}+\frac{\mu \nu}{1+\mu+\nu}+\alpha \sum_{i=1}^{p} \phi_{i} Z_{n+1-i}^{+} .
$$

Namely, we have

$$
\begin{aligned}
E\left(X_{n+1} \mid H_{n}\right) & =\sum_{i=1}^{p} \phi_{i} E\left(\alpha *_{n+1} X_{n+1-i}+\varepsilon_{n+1} \mid H_{n}\right) \\
& =\sum_{i=1}^{p} \phi_{i} E\left(\alpha *_{n+1} X_{n+1-i}+\varepsilon_{n+1} \mid Z_{n+1-i}\right) \\
& =(1-\alpha) \frac{\mu(1+\mu)}{1+\mu+\nu}+\frac{\mu \nu}{1+\mu+\nu}+\alpha \sum_{i=1}^{p} \phi_{i} Z_{n+1-i}^{+} .
\end{aligned}
$$


According to the previous results, it is implied that

$$
E\left(X_{n+1} \mid H_{n}\right)=\frac{\mu \nu}{1+\mu+\nu}+E\left(Z_{n+1}^{+} \mid H_{n}\right) .
$$

A more general statement can be proven,

$$
E\left(X_{n+k} \mid H_{n}\right)=\frac{\mu \nu}{1+\mu+\nu}+E\left(Z_{n+k}^{+} \mid H_{n}\right) .
$$

Using the properties of conditional mean and the fact that process history of the moment $n$ is contained in the process history of the moment $n+k-1$, we get

$$
\begin{aligned}
E\left(X_{n+k} \mid H_{n}\right) & =E\left(E\left(X_{n+k} \mid H_{n+k-1}\right) \mid H_{n}\right) \\
& =E\left(\frac{\mu \nu}{1+\mu+\nu}+E\left(Z_{n+k}^{+} \mid H_{n+k-1}\right) \mid H_{n}\right) \\
& =\frac{\mu \nu}{1+\mu+\nu}+E\left(E\left(Z_{n+k}^{+} \mid H_{n+k-1}\right) \mid H_{n}\right) \\
& =\frac{\mu \nu}{1+\mu+\nu}+E\left(Z_{n+k}^{+} \mid H_{n}\right) .
\end{aligned}
$$

It follows that $\operatorname{Cov}\left(X_{n+k}, Z_{n}\right)=\operatorname{Cov}\left(Z_{n+k}^{+}, Z_{n}\right)$, since

$$
\begin{aligned}
\operatorname{Cov}\left(X_{n+k}, Z_{n}\right) & =\operatorname{Cov}\left(E\left(X_{n+k} \mid Z_{n}\right), Z_{n}\right)=\operatorname{Cov}\left(E\left(E\left(X_{n+k} \mid H_{n}\right) \mid Z_{n}\right), Z_{n}\right) \\
& =\operatorname{Cov}\left(\frac{\mu \nu}{1+\mu+\nu}+E\left(E\left(Z_{n+k}^{+} \mid H_{n}\right) \mid Z_{n}\right), Z_{n}\right) \\
& =\operatorname{Cov}\left(E\left(E\left(Z_{n+k}^{+} \mid H_{n}\right) \mid Z_{n}\right), Z_{n}\right)=\operatorname{Cov}\left(Z_{n+k}^{+}, Z_{n}\right) .
\end{aligned}
$$

And due to the independence of series $\left\{X_{n}\right\}$ and $\left\{Y_{n}\right\}$, we have that $\operatorname{Cov}\left(X_{n+k}, Z_{n}\right)=$ $\operatorname{Cov}\left(X_{n+k}, X_{n}\right)$, which implies that $\operatorname{Cov}\left(Z_{n+k}^{+}, Z_{n}\right)=\operatorname{Cov}\left(X_{n+k}, X_{n}\right)$.

Analogous equations could be derived for a "negative" component $\left\{Y_{n}\right\}$,

$$
E\left(Y_{n+1} \mid H_{n}\right)=\frac{\mu \nu}{1+\mu+\nu}+E\left(Z_{n+1}^{-} \mid H_{n}\right) \text { and } \operatorname{Cov}\left(Y_{n+k}, Y_{n}\right)=-\operatorname{Cov}\left(Z_{n+k}^{-}, Z_{n}\right) \text {. }
$$

Regarding the correlation structure of the CGINAR(p) time series, shown in [16], the auto-covariance functions of the time series $\left\{X_{n}\right\}$ and $\left\{Y_{n}\right\}$ satisfy considered recurrent equations, so, the cross-covariance functions $\gamma_{Z Z^{+}}(k)$ and $\gamma_{Z Z^{-}}(k)$ do it too.

Since $C S D L I N A R(p)$ time series $\left\{Z_{n}\right\}$ is strictly stationary and ergodic, it could be proven that series $\left\{Z_{n}^{+}\right\}$and $\left\{Z_{n}^{-}\right\}$are strictly stationary and ergodic as well.

Proposition 3.6. Series $\left\{Z_{n}^{+}\right\}$and $\left\{Z_{n}^{-}\right\}$, defined as $Z_{n}^{+}=Z_{n} I_{\left\{Z_{n} \geq 0\right\}}$ and $Z_{n}^{-}=$ $Z_{n} I_{\left\{Z_{n}<0\right\}}$, where $\left\{Z_{n}\right\}$ is a CSDLINAR(p) time series, are strictly stationary and ergodic. 
Proof. Let $(\Omega, \mathcal{F}, P)$ be the probability space. The random variable $Z_{n}^{+}=$ $Z_{n} I_{\left\{Z_{n} \geq 0\right\}}$ could be presented as a function of a random variable $Z_{n}, Z_{n}^{+}=g\left(Z_{n}\right)=$ $\frac{\left|Z_{n}\right|+Z_{n}}{2}$. Regarding the fact that function $g(\cdot)$ is a composition of $\mathcal{F}$-measurable functions, it is $\mathcal{F}$-measurable as well. According to the Theorem 3.35 from [23], it follows that $Z_{n}^{+}$is strictly stationary and ergodic. Analogously, strong stationarity and ergodicity could be proven for the series $\left\{Z_{n}^{-}\right\}$.

Since covariance and cross-covariance functions satisfy shown recurrent relations, it follows that they exponentially tend to zero as lag size tends to infinity. Namely, if $\gamma_{p, \max }=\max _{0 \leq i \leq p}\left|\gamma_{Z Z^{+}}(i)\right|$, then $\left|\gamma_{Z Z^{+}}(k)\right| \leq \alpha^{j} \gamma_{p, \text { max }}, j p<k \leq(j+1) p$. This fact places our series into the class of asymptotically uncorrelated series. Also, from every state of the time series $\left\{Z_{n}\right\}$, it is possible, after a finite number of steps, to arrive to any other state. In other words, for any two positive integers $i$ and $j$, there exists a finite positive integer $m$ so that $P\left(Z_{n+m}=j \mid Z_{n}=i\right)>0$, i.e. time series $\left\{Z_{n}\right\}$ is irreducible. The time series $\left\{Z_{n}\right\}$ is aperiodic too, since from each state it is possible, after a finite, nonnegative number of steps, to come back to the same state, i.e. for any two integers $i$ and $m \geq 1, P\left(Z_{n+m}=i \mid Z_{n}=i\right)>0$. Regarding the Markovian property, irreducibility and aperiodicity, according to Theorem 3.2 from [4], the time series $\left\{Z_{n}\right\}$ is a strong mixing.

\section{Estimation of the unknown parameters}

To obtain the estimators of the unknown parameters of the $\operatorname{CSDLINAR(p)}$ model, we will, first, use the conditional least squares method. Let $\left(Z_{1}, Z_{2}, \cdots, Z_{N}\right)$ be a random sample of the $C S D L I N A R(p)$ process. The sum of squares that should be minimized is

$$
\begin{aligned}
Q_{N} & =\sum_{n=p+1}^{N}\left(Z_{n}-E\left(Z_{n} \mid H_{n-1}\right)\right)^{2} \\
& =\sum_{n=p+1}^{N}\left(Z_{n}-\left(\frac{(1-\alpha) \mu(1+\mu)}{1+\mu+\nu}-\frac{(1-\beta) \nu(1+\nu)}{1+\mu+\nu}+\alpha \sum_{i=1}^{p} \phi_{i} Z_{n-i}^{+}-\beta \sum_{i=1}^{p} \phi_{i} Z_{n-i}^{-}\right)\right)^{2} .
\end{aligned}
$$

If we take $\theta_{i}=\alpha \phi_{i}, \xi_{i}=\beta \phi_{i}, M^{+}=\frac{\mu(1+\mu)}{1+\mu+\nu}$ and $M^{-}=\frac{\nu(1+\nu)}{1+\mu+\nu}$, we get

$Q_{N}=\sum_{n=p+1}^{N}\left(Z_{n}-\left(\left(1-\sum_{i=1}^{p} \theta_{i}\right) M^{+}-\left(1-\sum_{i=1}^{p} \xi_{i}\right) M^{-}+\sum_{i=1}^{p} \theta_{i} Z_{n-i}^{+}-\sum_{i=1}^{p} \xi_{i} Z_{n-i}^{-}\right)\right)^{2}$.

The equations obtained by partial derivatives $\frac{\partial Q}{\partial M^{+}}$and $\frac{\partial Q}{\partial M^{-}}$are equivalent. That fact leads us to the conclusion that we will not have enough equations to solve the system. For that reason, let $M=\left(1-\sum_{i=1}^{p} \theta_{i}\right) M^{+}-\left(1-\sum_{i=1}^{p} \xi_{i}\right) M^{-}$. In that way, we will not be able to determine the estimators for $M^{+}$and $M^{-}$, i.e. for $\mu$ and $\nu$ but only for the expression $M=\frac{(1-\alpha) \mu(1+\mu)}{1+\mu+\nu}-\frac{(1-\beta) \nu(1+\nu)}{1+\mu+\nu}$. With this change, the 
sum of squares is

$$
Q_{N}=\sum_{n=p+1}^{N}\left(Z_{n}-\left(M+\sum_{i=1}^{p} \theta_{i} Z_{n-i}^{+}-\sum_{i=1}^{p} \xi_{i} Z_{n-i}^{-}\right)\right)^{2} .
$$

The system consisting of equations $\frac{\partial Q}{\partial M}=0, \frac{\partial Q}{\partial \theta_{j}}=0, \frac{\partial Q}{\partial \xi_{j}}=0, j=1, \ldots p$ is

$$
\begin{aligned}
M & =\bar{Z}^{(p)}-\sum_{i=1}^{p} \theta_{i} \bar{Z}^{+(p-i)}+\sum_{i=1}^{p} \xi_{i} \bar{Z}^{-(p-i)} \\
\hat{\gamma}_{Z Z^{+}}^{*}(p, p-i) & =\sum_{j=1}^{p} \theta_{j} \hat{\gamma}_{Z^{+} Z^{+}}^{*}(p-j, p-i)-\sum_{j=1}^{p} \xi_{j} \hat{\gamma}_{Z^{-} Z^{+}}^{*}(p-j, p-i), i=1,2, \cdots, p \\
\hat{\gamma}_{Z Z^{-}}^{*}(p, p-i) & =\sum_{j=1}^{p} \theta_{j} \hat{\gamma}_{Z^{+} Z^{-}}^{*}(p-j, p-i)-\sum_{j=1}^{p} \xi_{j} \hat{\gamma}_{Z^{-} Z^{-}}^{*}(p-j, p-i), i=1,2, \cdots, p,
\end{aligned}
$$

where the superscript $(k)$ refers to the sample mean of $N-p$ elements which are located after the $k$-th element of the sample,

$$
\bar{Z}^{(k)}=\frac{1}{N-p} \sum_{i=1}^{N-p} Z_{k+i}, \bar{Z}^{+(k)}=\frac{1}{N-p} \sum_{i=1}^{N-p} Z_{k+i}^{+}, \bar{Z}^{-(k)}=\frac{1}{N-p} \sum_{i=1}^{N-p} Z_{k+i}^{-}
$$

and $\hat{\gamma}_{Z^{\prime} Z^{\prime \prime}}^{*}(k, l)$ represents the sample cross-covariance of two subsequences of the series $\left\{Z_{n}^{\prime}\right\}$ and $\left\{Z_{n}^{\prime \prime}\right\}$, having length $N-p$, located after $k$-th and after $l$-th element, respectively, i.e.

$$
\hat{\gamma}_{Z^{\prime} Z^{\prime \prime}}^{\prime \prime}(k, l)=\frac{1}{N-p} \sum_{i=1}^{N-p}\left(Z_{k+i}^{\prime}-{\overline{Z^{\prime}}}^{(k)}\right)\left(Z_{l+i}^{\prime \prime}-{\overline{Z^{\prime \prime}}}^{(l)}\right) .
$$

Solving the system using Cramer's rule, we obtain that

$$
\hat{\theta}_{i}^{c l s}=\frac{D_{i}^{*}}{D^{*}}, \hat{\xi}_{i}^{c l s}=\frac{D_{p+i}^{*}}{D^{*}}, \quad i=1,2, \cdots, p, \quad \hat{M}^{c l s}=\bar{Z}^{(p)}-\sum_{i=1}^{p} \hat{\theta}_{i}^{c l s} \bar{Z}^{+(p-i)}+\sum_{i=1}^{p} \hat{\xi}_{i}^{c l s} \bar{Z}^{-(p-i)},
$$

where $D_{i}^{*}$ and $D^{*}$ are determinants of the Cramer's rule for the matrix of the system. Since $\sum_{i=1}^{p} \theta_{i}=\alpha, \sum_{i=1}^{p} \xi_{i}=\beta$ and $\phi_{i}=\frac{\theta_{i}}{\alpha}$ and also, $\phi_{i}=\frac{\xi_{i}}{\beta}$, we get

$$
\hat{\alpha}^{c l s}=\frac{\sum_{i=1}^{p} D_{i}^{*}}{D^{*}}, \hat{\beta}^{c l s}=\frac{\sum_{i=1}^{p} D_{p+i}^{*}}{D^{*}}, \hat{\phi}_{i}^{\prime c l s}=\frac{D_{i}^{*}}{\sum_{i=1}^{p} D_{i}^{*}}, \hat{\phi}_{i}^{\prime \prime c l s}=\frac{D_{p+i}^{*}}{\sum_{i=1}^{p} D_{p+i}^{*}}, \quad i=1,2, \cdots, p .
$$

Since we get, for each $\phi_{i}, i=1, \ldots, p$, two estimators, as the final estimator we can use the mean of these two, $\hat{\phi}_{i}^{\text {cls }}=\left(\hat{\phi}_{i}^{\prime c l s}+\hat{\phi}_{i}^{\prime \prime c l s}\right) / 2$.

In order to show a strong consistency and an asymptotic normality, first of estimators $\hat{M}^{\text {cls }}, \hat{\theta}_{i}^{c l s}$ and $\hat{\xi}_{i}^{c l s}$, and then of $\hat{\alpha}^{c l s}, \hat{\beta}^{c l s}, \hat{\phi}_{i}^{\text {'cls }}$ and $\hat{\phi}_{i}^{\prime \prime c l s}$, we will use 
the results from [21], the Theorems 3.1 and 3.2. Since the partial derivatives of the first order of $E\left(Z_{n} \mid H_{n-1}\right)$ are a constant and linear functions of $Z_{n-i}^{+}$and $Z_{n-i}^{-}$, the conditions $\mathrm{C} 1$ and $\mathrm{C} 3$ of the Theorem 3.1 are, obviously, satisfied. Regarding the condition $\mathrm{C} 2$, we have the following

$$
\begin{aligned}
& E\left(\left|\sum_{i=1}^{p}\left(a_{i} \frac{\partial E\left(Z_{n} \mid H_{n-1}\right)}{\partial \theta_{i}}+a_{i+p} \frac{\partial E\left(Z_{n} \mid H_{n-1}\right)}{\partial \xi_{i}}\right)+a_{2 p+1} \frac{\partial E\left(Z_{n} \mid H_{n-1}\right)}{\partial M}\right|^{2}\right)=0 \\
& \Rightarrow a_{i}=0, i=1,2, \ldots 2 p+1 .
\end{aligned}
$$

But,

$$
\begin{aligned}
& E\left(\left|\sum_{i=1}^{p}\left(a_{i} \frac{\partial E\left(Z_{n} \mid H_{n-1}\right)}{\partial \theta_{i}}+a_{i+p} \frac{\partial E\left(Z_{n} \mid H_{n-1}\right)}{\partial \xi_{i}}\right)+a_{2 p+1} \frac{\partial E\left(Z_{n} \mid H_{n-1}\right)}{\partial M}\right|^{2}\right) \\
& =E\left(\left|\sum_{i=1}^{p}\left(a_{i} Z_{n-i}^{+}-a_{i+p} Z_{n-i}^{-}\right)+a_{2 p+1}\right|^{2}\right) .
\end{aligned}
$$

If we add and subtract the expected value of the variable $T \equiv \sum_{i=1}^{p}\left(a_{i} Z_{n-i}^{+}-a_{i+p} Z_{n-i}^{-}\right)$, we get that

$$
E\left(\left|\sum_{i=1}^{p}\left(a_{i} Z_{n-i}^{+}-a_{i+p} Z_{n-i}^{-}\right)+a_{2 p+1}\right|^{2}\right)=E\left(|T-E T+c|^{2}\right)
$$

where $c=a_{2 p+1}+E T$. Next, we get

$$
E\left(|T-E T+c|^{2}\right)=\operatorname{Var}(T)+2 c E(T-E T)+c^{2}=\operatorname{Var}(T)+c^{2} .
$$

The equality of the last expression to zero implies that $\operatorname{Var}(T)=0$ and $c=0$. In the case of the time series $\left\{Z_{n}\right\}$, we will show that $\operatorname{Var}(T)=0$ implies that $a_{i}=0$, $1 \leq i \leq 2 p$.

The fact that $\operatorname{Var}(T)=0$ means that $T=$ const, almost sure, which implies that random variables $Z_{n-1}^{+}, \ldots, Z_{n-p}^{+}, Z_{n-1}^{-}, \ldots, Z_{n-p}^{-}$are linearly dependent, except in the case when $T=0$ and $a_{i}=0,1 \leq i \leq 2 p$. Now, we will show that the mentioned exception is the only possible case. Let us focus on the covariance matrix of the vector of random variables $\left[Z_{n-1}^{+}, Z_{n-1}^{-}, Z_{n-2}^{+}, Z_{n-2}^{-}, \ldots, Z_{n-p}^{+}, Z_{n-p}^{-}\right]^{T}$. On its diagonal there are variances, which are greater than zero. Secondly, the covariances between the elements of the random vector are $\gamma_{Z^{+}}(k), \gamma_{Z^{-}}(k), \gamma_{Z^{+} Z^{-}}(k)$ and $\gamma_{Z^{-} Z^{+}}(k)$ which all exponentially tend to zero, when $k$ tends to infinity. According to the Proposition 5.1.1 from [6], these two facts ensure that covariance matrix is regular. The regularity of the covariance matrix excludes linear dependence between variables, so the only possible case is $T=0$ and $a_{i}=0,1 \leq i \leq 2 p$. With the fact that $c=0$, we have that $a_{2 p+1}=0$ and we have all three conditions of the Theorem 3.1 from [21] fulfilled. Hence, the estimators $\hat{\theta}_{i}^{c l s}, \hat{\xi}_{i}^{c l s}, i=1, \ldots, p$ and $\hat{M}^{c l s}$ are strongly consistent. 
Regarding the asymptotic normality of the estimators, we should consider the Theorem 3.2 from [21]. Since function $f_{n \mid n-1}=\operatorname{Var}\left(Z_{n} \mid H_{n-1}\right)$, the condition D1 demands all the elements of the matrix

$$
\begin{aligned}
R \equiv E( & \left(Z_{n-1}^{+}, Z_{n-2}^{+}, \ldots, Z_{n-p}^{+}, Z_{n-1}^{-}, Z_{n-2}^{-}, \ldots, Z_{n-p}^{-}, 1\right)^{T} \operatorname{Var}\left(Z_{n} \mid H_{n-1}\right) \\
& \left.\times\left(Z_{n-1}^{+}, Z_{n-2}^{+}, \ldots, Z_{n-p}^{+}, Z_{n-1}^{-}, Z_{n-2}^{-}, \ldots, Z_{n-p}^{-}, 1\right)\right)
\end{aligned}
$$

to be finite. To prove that, it would be enough to show that for join moments stands $E\left(A^{k_{1}} B^{k_{2}} C^{k_{3}} D^{k_{4}}\right)<\infty$, where random variables $A, B, C$ and $D$ could be $Z_{n-i}^{+}$or $Z_{n-i}^{-}, 1 \leq i \leq p$ and $k_{1}, k_{2}, k_{3}, k_{4} \in\{0,1,2,3,4\}$. First, we will show that $E\left(Z_{n-i}^{+}\right)^{k}<\infty, k \geq 0$. Using the definition of the random variable $Z_{n-i}^{+}$, we have

$$
E\left(Z_{n-i}^{+}\right)^{k}=\frac{1}{1+\mu+\nu} \sum_{x=0}^{\infty} x^{k}\left(\frac{\mu}{1+\mu}\right)^{x}<\infty
$$

Analogously, for the random variable $Z_{n-i}^{-}$we conclude the same. Next, using the Cauchy-Schwarz inequality, we have

$$
\begin{aligned}
E\left(A^{k_{1}} B^{k_{2}} C^{k_{3}} D^{k_{4}}\right) & \leq \sqrt{E\left(A^{2 k_{1}} B^{2 k_{2}}\right) E\left(C^{2 k_{3}} D^{2 k_{4}}\right)} \\
& \leq \sqrt{\sqrt{E\left(A^{4 k_{1}}\right) E\left(B^{4 k_{2}}\right)} \sqrt{E\left(C^{4 k_{3}}\right) E\left(D^{4 k_{4}}\right)}} \\
& =\sqrt[4]{E\left(A^{4 k_{1}}\right) E\left(B^{4 k_{2}}\right) E\left(C^{4 k_{3}}\right) E\left(D^{4 k_{4}}\right)}<\infty .
\end{aligned}
$$

It means, according to the Theorem 3.2 from [21], that the vector of estimates $\tilde{\theta}_{N}^{c l s} \equiv\left(\hat{\theta}_{1}^{c l s}, \ldots, \hat{\theta}_{p}^{c l s}, \hat{\xi}_{1}^{c l s}, \ldots, \hat{\xi}_{p}^{c l s}, \hat{M}^{c l s}\right)^{T}$ is asymptotically normally distributed,

$$
N^{-1 / 2}\left(\tilde{\theta}_{N}^{c l s}-\theta^{\prime}\right) \stackrel{d}{\rightarrow} \mathcal{N}\left(\mathbf{0}, U^{-1} R U^{-1}\right)
$$

where $\theta^{\prime}=\left(\theta_{1}, \ldots, \theta_{p}, \xi_{1}, \ldots, \xi_{p}, M\right)^{T}$ and $U$ is a block matrix

$$
U=E\left[\begin{array}{c|c|c}
Z_{n-i}^{+} Z_{n-j}^{+} & Z_{n-i}^{+} Z_{n-j}^{-} & Z_{n-i}^{+} \\
\hline Z_{n-i}^{-} Z_{n-j}^{+} & Z_{n-i}^{-} Z_{n-j}^{-} & Z_{n-i}^{-} \\
\hline Z_{n-i}^{+} & Z_{n-j}^{-} & 1
\end{array}\right], 1 \leq i, j \leq p
$$

Let us, now, define a function

$$
\mathbf{g}\left(x_{1}, x_{2}, \ldots, x_{2 p+1}\right)=\left(g_{1}\left(x_{1}, x_{2}, \ldots, x_{2 p+1}\right), \ldots, g_{p+3}\left(x_{1}, x_{2}, \ldots, x_{2 p+1}\right)\right)
$$


with coordinate functions:

$$
\begin{aligned}
g_{1}\left(x_{1}, x_{2}, \ldots, x_{2 p+1}\right) & =\sum_{i=1}^{p} x_{i} \\
g_{2}\left(x_{1}, x_{2}, \ldots, x_{2 p+1}\right) & =\sum_{i=1}^{p} x_{p+i} \\
g_{2+j}\left(x_{1}, x_{2}, \ldots, x_{2 p+1}\right) & =\frac{1}{2}\left(\frac{x_{j}}{\sum_{i=1}^{p} x_{i}}+\frac{x_{p+j}}{\sum_{i=1}^{p} x_{p+i}}\right), j=1, \ldots p, \\
g_{p+3}\left(x_{1}, x_{2}, \ldots, x_{2 p+1}\right) & =x_{2 p+1} .
\end{aligned}
$$

Since $\mathbf{g}\left(\hat{\theta}_{1}^{c l s}, \ldots, \hat{\theta}_{p}^{c l s}, \hat{\xi}_{1}^{c l s}, \ldots, \hat{\xi}_{p}^{c l s}, \hat{M}^{c l s}\right)=\left(\hat{\alpha}^{c l s}, \hat{\beta}^{c l s}, \hat{\phi}_{1}^{c l s}, \ldots, \hat{\phi}_{p}^{c l s}, \hat{M}^{c l s}\right)$ and the function $\mathbf{g}$ is continuous, it implies that estimators $\hat{\alpha}^{c l s}, \hat{\beta}^{c l s}, \hat{\phi}_{i}^{c l s}, i=1, \ldots p$ and $\hat{M}^{c l s}$ are strongly consistent. Also, the function $\mathbf{g}$ satisfies conditions of the Proposition 6.4.3 from [6], hence, estimators $\hat{\alpha}^{c l s}, \hat{\beta}^{c l s}, \hat{\phi}_{i}^{c l s}, i=1, \ldots p$ and $\hat{M}^{c l s}$, have an asymptotic normal distribution too. After all, we have the following theorem.

Theorem 4.1. Statistics obtained by the conditional least squares method, $\hat{\alpha}^{c l s,}$ $\hat{\beta}^{c l s}, \hat{\phi}_{i}^{c l s}, i=1, \ldots p$ and $\hat{M}^{\text {cls }}$ are strongly consistent and asymptotically normal estimators of corresponding parameters of the CSDLINAR $(p)$ model,

$$
N^{-1 / 2}\left(\hat{\theta}_{N}^{c l s}-\theta\right) \stackrel{d}{\rightarrow} \mathcal{N}\left(\mathbf{0}, J U^{-1} R U^{-1} J^{T}\right),
$$

where $\hat{\theta}_{N}^{c l s}=\left(\hat{\alpha}^{c l s}, \hat{\beta}^{c l s}, \hat{\phi}_{1}^{c l s}, \ldots, \hat{\phi}_{p}^{c l s}, \hat{M}^{c l s}\right)^{T}, \theta=\left(\alpha, \beta, \phi_{1}, \ldots, \phi_{p}, M\right)^{T}$ and matrix $J$ is the Jacobian of mapping $\mathbf{g}$.

Now, as the method of estimation of unknown parameters $\mu, \nu, \alpha, \beta$ and $\phi_{i}, i=$ $1,2, \ldots, p$ we use the Yule-Walker method. For estimating parameters $\mu$ and $\nu$ we use the mean and the variance of the $C S D L I N A R(p)$ time series $\left\{Z_{n}\right\}$. The equations $\bar{Z}_{n}=\mu-\nu$ and $\hat{\gamma}_{Z}(0)=\mu(1+\mu)+\nu(1+\nu)$ give the following statistics.

$$
\begin{aligned}
& \hat{\mu}^{Y W}=-\frac{1}{2}+\frac{1}{2} \bar{Z}_{N}+\frac{1}{2} \sqrt{1-\bar{Z}_{N}^{2}+2 \hat{\gamma}_{Z}(0)} \\
& \hat{\nu}^{Y W}=-\frac{1}{2}-\frac{1}{2} \bar{Z}_{N}+\frac{1}{2} \sqrt{1-\bar{Z}_{N}^{2}+2 \hat{\gamma}_{Z}(0)} .
\end{aligned}
$$

To obtain estimators of parameters $\alpha, \beta$ and $\phi_{i}, i=1,2, \ldots, p$, we use the systems of equations

$$
\hat{\gamma}_{Z^{+}}(k)=\sum_{i=1}^{p} \alpha \phi_{i} \hat{\gamma}_{Z^{+}}(k-i), k=1,2, \ldots, p \quad \text { and } \quad \hat{\gamma}_{Z^{-}}(k)=\sum_{i=1}^{p} \beta \phi_{i} \hat{\gamma}_{Z^{-}}(k-i), k=1,2, \ldots, p .
$$

Replacing $\alpha \phi_{i}$ with $\theta_{i}$ and $\beta \phi_{i}$ with $\xi_{i}$, using the fact that $\sum_{i=1}^{p} \theta_{i}=\alpha$ and $\sum_{i=1}^{p} \xi_{i}=\beta$ and applying Cramer's rule, we get the solution:

$$
\hat{\theta}_{i}^{Y W}=\frac{D_{A_{i}}}{D}, \hat{\xi}_{i}^{Y W}=\frac{D_{B_{i}}}{D} \text { or }
$$




$$
\begin{array}{r}
\hat{\alpha}^{Y W}=\frac{\sum_{i=1}^{p} D_{A_{i}}}{D}, \hat{\beta}^{Y W}=\frac{\sum_{i=1}^{p} D_{B_{i}}}{D} \text { and } \\
\hat{\phi}_{i}^{\prime Y W}=\frac{D_{A_{i}}}{\sum_{i=1}^{p} D_{A_{i}}} \text { and } \hat{\phi}_{i}^{\prime \prime Y W}=\frac{D_{B_{i}}}{\sum_{i=1}^{p} D_{B_{i}}}, i=1,2, \ldots, p,
\end{array}
$$

where $D, D_{A_{i}}, D_{B_{i}}$ are appropriate determinants used in Cramer's rule. Again, as the final estimation of parameter $\phi_{i}$ we will use the mean of $\hat{\phi}_{i}^{\prime Y W}$ and $\hat{\phi}_{i}^{\prime \prime Y W}$, $\hat{\phi}_{i}^{Y W}=\left(\hat{\phi}_{i}^{\prime Y W}+\hat{\phi}_{i}^{\prime \prime Y W}\right) / 2$.

The obtained determinants are polynomials of covariance and cross-covariance functions, so, the obtained estimators are rational functions of covariance and crosscovariance functions. Since the time series $\left\{Z_{n}\right\}$ is ergodic and strictly stationary and, according to the Theorems 3.34 and 3.35 from [23], sample covariations are strongly consistent estimators of corresponding covariations. As rational functions of strongly consistent estimators, statistics $\hat{\theta}_{i}^{Y W}, \hat{\xi}_{i}^{Y W}$ and $\hat{\phi}_{i}^{Y W}, 1 \leq i \leq p$, are strongly consistent too. In the same way, as it is done in [20], it could be shown that sample autocovariance and cross-covariance functions of time series $\left\{Z_{n}\right\},\left\{Z_{n}^{+}\right\}$ and $\left\{Z_{n}^{-}\right\}$have an asymptotically normal distribution. It means that

$$
N^{-1 / 2}\left(\tilde{\theta}_{N}^{Y W}-\theta^{\prime \prime}\right) \stackrel{d}{\rightarrow} \mathcal{N}\left(\mathbf{0}, C V C^{T}\right),
$$

where the vector of statistics $\tilde{\theta}_{N}^{Y W}=\left(\hat{\theta}_{1}^{Y W}, \ldots, \hat{\theta}_{p}^{Y W}, \hat{\xi}_{1}^{Y W}, \ldots, \hat{\xi}_{p}^{Y W}, \bar{Z}_{N}, \hat{\gamma}_{Z}(0)\right)^{T}$, vector of parameters $\theta^{\prime \prime}=\left(\theta_{1}, \ldots, \theta_{p}, \xi_{1}, \ldots, \xi_{p}, E Z_{n}, \gamma_{Z}(0)\right)^{T}$, $\mathrm{V}$ is block matrix

$$
V=\left[\begin{array}{cccc}
{\left[V_{11}\right]_{1 \times 1}} & {\left[V_{12}\right]_{1 \times 1}} & {\left[V_{13}\right]_{1 \times(p+1)}} & {\left[V_{14}\right]_{1 \times(p+1)}} \\
{\left[V_{12}\right]_{1 \times 1}} & {\left[V_{22}\right]_{1 \times 1}} & {\left[V_{23}\right]_{1 \times(p+1)}} & {\left[V_{24}\right]_{1 \times(p+1)}} \\
{\left[V_{13}\right]_{1 \times(p+1)}^{T}} & {\left[V_{23}\right]_{1 \times(p+1)}^{T}} & {\left[V_{33}\right]_{(p+1) \times(p+1)}} & {\left[V_{34}\right]_{(p+1) \times(p+1)}} \\
{\left[V_{14}\right]_{1 \times(p+1)}^{T}} & {\left[V_{24}\right]_{1 \times(p+1)}^{T}} & {\left[V_{34}\right]_{(p+1) \times(p+1)}^{T}} & {\left[V_{44}\right]_{(p+1) \times(p+1)}}
\end{array}\right],
$$

where

$$
\begin{array}{ll}
{\left[V_{11}\right]=\lim _{N \rightarrow \infty} N \operatorname{Var}\left(\bar{Z}_{N}\right)} & {\left[V_{23}\right]_{1, j}=\lim _{N \rightarrow \infty} N \operatorname{Cov}\left(\hat{\gamma}_{Z}(0), \hat{\gamma}_{Z^{+}}(j-1)\right)} \\
{\left[V_{12}\right]=\lim _{N \rightarrow \infty} N \operatorname{Cov}\left(\bar{Z}_{N}, \hat{\gamma}_{Z}(0)\right)} & {\left[V_{24}\right]_{1, j}=\lim _{N \rightarrow \infty} N \operatorname{Cov}\left(\hat{\gamma}_{Z}(0), \hat{\gamma}_{Z^{-}}(j-1)\right)} \\
{\left[V_{13}\right]_{1, j}=\lim _{N \rightarrow \infty} N \operatorname{Cov}\left(\bar{Z}_{N}, \hat{\gamma}_{Z^{+}}(j-1)\right)} & {\left[V_{33}\right]_{i, j}=\lim _{N \rightarrow \infty} N \operatorname{Cov}\left(\hat{\gamma}_{Z^{+}}(i-1), \hat{\gamma}_{Z^{+}}(j-1)\right)} \\
{\left[V_{14}\right]_{1, j}=\lim _{N \rightarrow \infty} N \operatorname{Cov}\left(\bar{Z}_{N}, \hat{\gamma}_{Z^{-}}(j-1)\right)} & {\left[V_{34}\right]_{i, j}=\lim _{N \rightarrow \infty} N \operatorname{Cov}\left(\hat{\gamma}_{Z^{+}}(i-1), \hat{\gamma}_{Z^{-}}(j-1)\right)} \\
{\left[V_{22}\right]=\lim _{N \rightarrow \infty} N \operatorname{Var}\left(\hat{\gamma}_{Z}(0)\right)} & {\left[V_{44}\right]_{i, j}=\lim _{N \rightarrow \infty} N \operatorname{Cov}\left(\hat{\gamma}_{Z^{-}}(i-1), \hat{\gamma}_{Z^{-}}(j-1)\right)}
\end{array}
$$

where $1 \leq i, j \leq p+1$ and matrix $C$ is the Jacobian of mapping which transforms sample covariances, by Cramer's rule, i.e. equations (4.1), into statistics $\hat{\theta}_{i}^{Y W}, \hat{\xi}_{i}^{Y W}$, $1 \leq i \leq p$.

Then, using the function

$$
\mathbf{g}^{*}\left(x_{1}, x_{2}, \ldots, x_{2 p+2}\right)=\left(g_{1}^{*}\left(x_{1}, x_{2}, \ldots, x_{2 p+2}\right), \ldots, g_{p+4}^{*}\left(x_{1}, x_{2}, \ldots, x_{2 p+2}\right)\right)
$$


with coordinate functions:

$$
\begin{aligned}
g_{1}^{*}\left(x_{1}, x_{2}, \ldots, x_{2 p+2}\right) & =\sum_{i=1}^{p} x_{i}, \\
g_{2}^{*}\left(x_{1}, x_{2}, \ldots, x_{2 p+2}\right) & =\sum_{i=1}^{p} x_{p+i}, \\
g_{2+j}^{*}\left(x_{1}, x_{2}, \ldots, x_{2 p+2}\right) & =\frac{1}{2}\left(\frac{x_{j}}{\sum_{i=1}^{p} x_{i}}+\frac{x_{p+j}}{\sum_{i=1}^{p} x_{p+i}}\right), j=1, \ldots p, \\
g_{p+3}^{*}\left(x_{1}, x_{2}, \ldots, x_{2 p+2}\right) & =-\frac{1}{2}+\frac{1}{2} x_{2 p+1}+\frac{1}{2} \sqrt{1-x_{2 p+1}^{2}+2 x_{2 p+2}}, \\
g_{p+4}^{*}\left(x_{1}, x_{2}, \ldots, x_{2 p+2}\right) & =-\frac{1}{2}-\frac{1}{2} x_{2 p+1}+\frac{1}{2} \sqrt{1-x_{2 p+1}^{2}+2 x_{2 p+2}},
\end{aligned}
$$

we map the vector of estimators $\left(\hat{\theta}_{1}^{Y W}, \ldots, \hat{\theta}_{p}^{Y W}, \hat{\xi}_{1}^{Y W}, \ldots, \hat{\xi}_{p}^{Y W}, \bar{Z}, \hat{\gamma}_{Z}(0)\right)$ to the vector $\left(\hat{\alpha}^{Y W}, \hat{\beta}^{Y W}, \hat{\phi}_{1}^{Y W}, \ldots, \hat{\phi}_{p}^{Y W}, \hat{\mu}^{Y W}, \hat{\nu}^{Y W}\right)$. According to the Proposition 6.4.3 from [6], estimators $\hat{\mu}^{Y W}, \hat{\nu}^{Y W}, \hat{\alpha}^{Y W}, \hat{\beta}^{Y W}$ and $\hat{\phi}_{i}^{Y W}, i=1,2, \ldots, p$ are asymptotically normal too. Speaking in the form of a theorem, we have the following.

Theorem 4.2. Statistics obtained by the Yule-Walker method, $\hat{\mu}^{Y W}, \hat{\nu}^{Y W}, \hat{\alpha}^{Y W}$, $\hat{\beta}^{Y W}$ and $\hat{\phi}_{i}^{Y W}, i=1, \ldots p$ are strongly consistent and asymptotically normal estimators of corresponding parameters of the CSDLINAR $(p)$ model,

$$
N^{-1 / 2}\left(\hat{\theta}_{N}^{Y W}-\theta\right) \stackrel{d}{\rightarrow} \mathcal{N}\left(\mathbf{0}, G C V C^{T} G^{T}\right),
$$

where the vector $\hat{\theta}_{N}^{Y W}=\left(\hat{\mu}^{Y W}, \hat{\nu}^{Y W}, \hat{\alpha}^{Y W}, \hat{\beta}^{Y W}, \hat{\phi}_{1}^{Y W}, \ldots, \hat{\phi}_{p}^{c l s}, \hat{M}^{\text {cls }}\right)^{T}$ and the vector $\theta=\left(\alpha, \beta, \phi_{1}, \ldots, \phi_{p}, M\right)^{T}$ and matrix $G$ is the Jacobian of mapping $\mathbf{g}^{*}$.

\section{Simulations, real data application, identification and prediction of latent components}

In this section some practical results will be presented. First, in order to illustrate the influence of the parameters to the form of the time series and asymptotic behavior of the obtained estimators, for different true values of the the parameters, we simulated 1000 samples and then we calculated estimators and some quantifiers of the estimation quality. Since in the subsection with real data application it turned out that the model of order three was the most adequate, we chose to simulate the model of order three. Then, we will present application on real data and comparison with other models. Last but not least, an interesting application will be shown. Since skew discrete Laplace distribution can be presented as a difference of two geometrically distributed components, we try to identify and predict these two opposite latent factors influencing our process. 


\subsection{Simulations}

To confirm the conclusions about the asymptotic properties of the obtained estimators, we simulate, for different true values of model parameters, 1000 series of $C S D L I N A R(3)$ time series, each of size $\mathrm{N}=5000$. Simulations were performed using the Monte Carlo method. For simulations, the fact that the CSDLINAR(p) process can be presented as a difference of two $C G I N A R(p)$ processes was used. First, for both $C G I N A R$ series, three initial values are generated as realizations of geometrically distributed random variables. Then, using the definition of the thinning operator $(\alpha, \beta) \odot_{m}$, the rest of the sequence was generated. In the end, the CSDLINAR(3) time series was obtained as the difference of generated CGINAR series. The values of true parameters were chosen in order to demonstrate their influence on the process behavior. On the figures 5.1 and 5.2 trajectories and values of the auto correlation functions of four CSDLINAR(3) models with different parameters are shown. The conditional least square and Yule-Walker methods
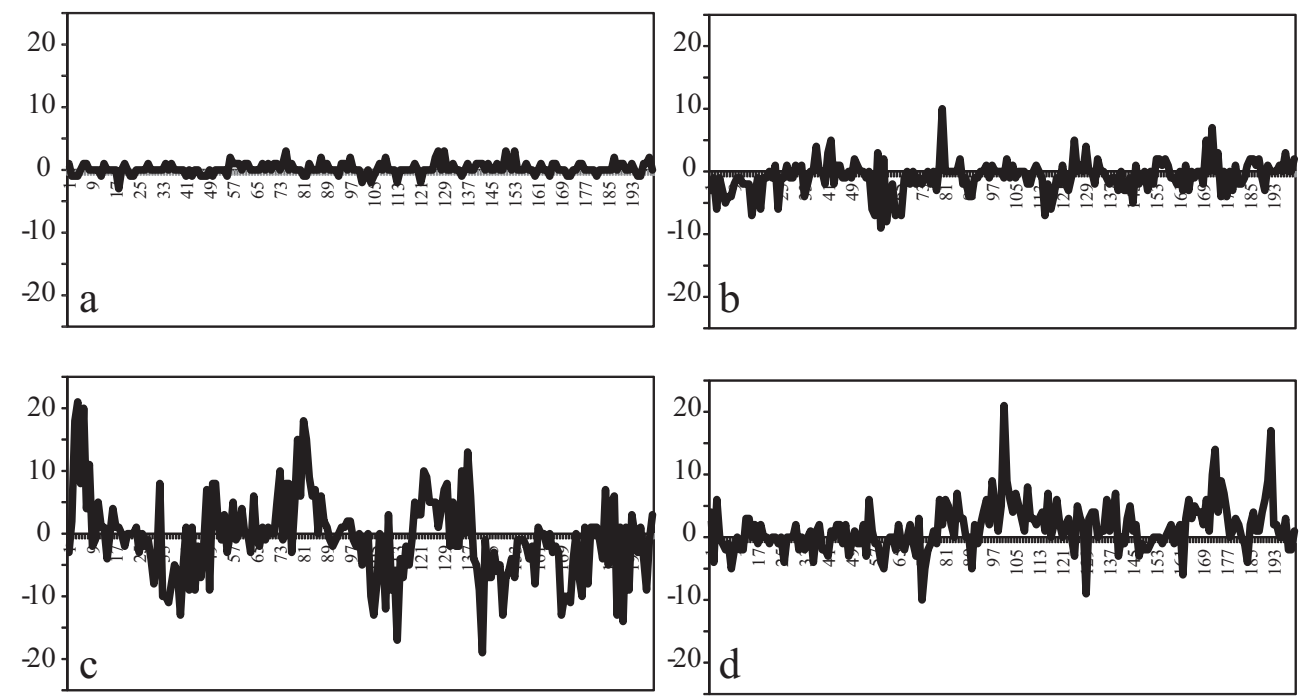

FIG. 5.1: Trajectories of simulated time series with $\mu=0.5, \nu=0.2, \alpha=0.3$, $\beta=0.1$ (a), $\mu=1, \nu=2, \alpha=0.3, \beta=0.5$ (b), $\mu=4, \nu=10, \alpha=0.7, \beta=0.9$ (c) and $\mu=8, \nu=2, \alpha=0.8, \beta=0.4(\mathrm{~d})$

are used to obtain parameter estimates. Unfortunately, by the conditional least square method we couldn't obtain the estimates for parameters $\mu$ and $\nu$ but only for their function $M=(1-\alpha) \frac{\mu(1+\mu)}{1+\mu+\nu}+(1-\beta) \frac{\nu(1+\nu)}{1+\mu+\nu}$. To illustrate stationarity of the estimators, for each model, samples of four different sizes are used, 500, 1000, 3000 and 5000. The results of estimation are shown in Tables 5.1 and 5.2.

Obviously, both methods give estimators that converge to the true values of parameters. Regarding precision and stability of the estimators, we could say that, in case of parameters $\alpha$ and $\beta$, estimators obtained by the conditional least squares 
Table 5.1: Realizations of estimators for parameters $\mu$ and $\nu$ and their standard deviations (in parentheses)

\begin{tabular}{|c|c|c|c|c|c|c|}
\hline$\mu=0.5$ & $\nu=0.2$ & $\alpha=0.3$ & $\beta=0.1$ & $\phi_{1}=0.2$ & $\phi_{2}=0.2$ & $\phi_{3}=0.6$ \\
\hline $\bar{N}$ & $\hat{\mu}^{Y W}$ & $\hat{\nu}^{Y W}$ & $\hat{\alpha}^{c l s}$ & $\hat{\alpha}^{Y W}$ & $\hat{\beta}^{c l s}$ & $\hat{\beta}^{Y W}$ \\
\hline 500 & $\begin{array}{c}0.499 \\
(0.058)\end{array}$ & $\begin{array}{c}0.197 \\
(0.035)\end{array}$ & $\begin{array}{c}0.288 \\
(0.100)\end{array}$ & $\begin{array}{c}0.265 \\
(0.086)\end{array}$ & $\begin{array}{c}0.082 \\
(0.184)\end{array}$ & $\begin{array}{c}0.079 \\
(0.088)\end{array}$ \\
\hline 1000 & $\begin{array}{c}0.500 \\
(0.041)\end{array}$ & $\begin{array}{c}0.199 \\
(0.025)\end{array}$ & $\begin{array}{c}0.296 \\
(0.071)\end{array}$ & $\begin{array}{c}0.273 \\
(0.062)\end{array}$ & $\begin{array}{c}0.091 \\
(0.130)\end{array}$ & $\begin{array}{c}0.085 \\
(0.063)\end{array}$ \\
\hline 3000 & $\begin{array}{c}0.500 \\
(0.025)\end{array}$ & $\begin{array}{c}0.199 \\
(0.015)\end{array}$ & $\begin{array}{c}0.299 \\
(0.042)\end{array}$ & $\begin{array}{c}0.278 \\
(0.037)\end{array}$ & $\begin{array}{c}0.097 \\
(0.075)\end{array}$ & $\begin{array}{c}0.086 \\
(0.037)\end{array}$ \\
\hline 5000 & $\begin{array}{c}0.501 \\
(0.019)\end{array}$ & $\begin{array}{c}0.199 \\
(0.011)\end{array}$ & $\begin{array}{c}0.300 \\
(0.033)\end{array}$ & $\begin{array}{c}0.279 \\
(0.029)\end{array}$ & $\begin{array}{c}0.097 \\
(0.058)\end{array}$ & $\begin{array}{c}0.087 \\
(0.028)\end{array}$ \\
\hline$\mu=1$ & $\nu=2$ & $\alpha=0.3$ & $\beta=0.5$ & $\phi_{1}=0.1$ & $\phi_{2}=0.7$ & $\phi_{3}=0.2$ \\
\hline$N$ & $\hat{\mu}^{Y W}$ & $\hat{\nu}^{Y W}$ & $\hat{\alpha}^{c l s}$ & $\hat{\alpha}^{Y W}$ & $\hat{\beta}^{c l s}$ & $\hat{\beta}^{Y W}$ \\
\hline 500 & $\begin{array}{c}0.990 \\
(0.131)\end{array}$ & $\begin{array}{c}1.984 \\
(0.212)\end{array}$ & $\begin{array}{c}0.278 \\
(0.193)\end{array}$ & $\begin{array}{c}0.256 \\
(0.088)\end{array}$ & $\begin{array}{c}0.486 \\
(0.097)\end{array}$ & $\begin{array}{c}0.443 \\
(0.081)\end{array}$ \\
\hline 1000 & $\begin{array}{c}0.997 \\
(0.096)\end{array}$ & $\begin{array}{c}1.995 \\
(0.150)\end{array}$ & $\begin{array}{c}0.286 \\
(0.130)\end{array}$ & $\begin{array}{c}0.263 \\
(0.063)\end{array}$ & $\begin{array}{c}0.496 \\
(0.068)\end{array}$ & $\begin{array}{c}0.454 \\
(0.059)\end{array}$ \\
\hline 3000 & $\begin{array}{c}0.998 \\
(0.056)\end{array}$ & $\begin{array}{c}1.996 \\
(0.085)\end{array}$ & $\begin{array}{c}0.293 \\
(0.078)\end{array}$ & $\begin{array}{c}0.269 \\
(0.039)\end{array}$ & $\begin{array}{c}0.502 \\
(0.041)\end{array}$ & $\begin{array}{c}0.459 \\
(0.034)\end{array}$ \\
\hline 5000 & $\begin{array}{c}0.999 \\
(0.043)\end{array}$ & $\begin{array}{c}1.997 \\
(0.066)\end{array}$ & $\begin{array}{c}0.294 \\
(0.060)\end{array}$ & $\begin{array}{c}0.269 \\
(0.030)\end{array}$ & $\begin{array}{c}0.502 \\
(0.032)\end{array}$ & $\begin{array}{c}0.460 \\
(0.027)\end{array}$ \\
\hline$\mu=4$ & $\nu=10$ & $\alpha=0.7$ & $\beta=0.9$ & $\phi_{1}=0.3$ & $\phi_{2}=0.4$ & $\phi_{3}=0.3$ \\
\hline$N$ & $\hat{\mu}^{Y W}$ & $\hat{\nu}^{Y W}$ & $\hat{\alpha}^{\mathrm{cls}}$ & $\hat{\alpha}^{Y W}$ & $\hat{\beta}^{c l s}$ & $\hat{\beta}^{Y W}$ \\
\hline 500 & $\begin{array}{c}3.463 \\
(1.141)\end{array}$ & $\begin{array}{c}9.551 \\
(2.477)\end{array}$ & $\begin{array}{c}0.665 \\
(0.173)\end{array}$ & $\begin{array}{c}0.641 \\
(0.098)\end{array}$ & $\begin{array}{c}0.874 \\
(0.058)\end{array}$ & $\begin{array}{c}0.848 \\
(0.056)\end{array}$ \\
\hline 1000 & $\begin{array}{c}3.732 \\
(0.879)\end{array}$ & $\begin{array}{c}9.791 \\
(1.875)\end{array}$ & $\begin{array}{c}0.681 \\
(0.107)\end{array}$ & $\begin{array}{c}0.662 \\
(0.067)\end{array}$ & $\begin{array}{c}0.888 \\
(0.040)\end{array}$ & $\begin{array}{c}0.865 \\
(0.038)\end{array}$ \\
\hline 3000 & $\begin{array}{c}3.926 \\
(0.548)\end{array}$ & $\begin{array}{c}9.925 \\
(1.149)\end{array}$ & $\begin{array}{c}0.688 \\
(0.058)\end{array}$ & $\begin{array}{c}0.676 \\
(0.038)\end{array}$ & $\begin{array}{c}0.900 \\
(0.022)\end{array}$ & $\begin{array}{c}0.878 \\
(0.022)\end{array}$ \\
\hline 5000 & $\begin{array}{c}3.949 \\
(0.425)\end{array}$ & $\begin{array}{c}9.935 \\
(0.889)\end{array}$ & $\begin{array}{c}0.689 \\
(0.046)\end{array}$ & $\begin{array}{c}0.679 \\
(0.030)\end{array}$ & $\begin{array}{c}0.902 \\
(0.017)\end{array}$ & $\begin{array}{c}0.880 \\
(0.017)\end{array}$ \\
\hline$\mu=6$ & $\nu=2$ & $\alpha=0.8$ & $\beta=0.4$ & $\phi_{1}=0.5$ & $\phi_{2}=0.33$ & $\phi_{3}=0.17$ \\
\hline$N$ & $\hat{\mu}^{Y W}$ & $\hat{\nu}^{Y W}$ & $\hat{\alpha}^{c l s}$ & $\hat{\alpha}^{Y W}$ & $\hat{\beta}^{c l s}$ & $\hat{\beta}^{Y W}$ \\
\hline 500 & $\begin{array}{c}5.875 \\
(1.043)\end{array}$ & $\begin{array}{c}1.859 \\
(0.520)\end{array}$ & $\begin{array}{c}0.786 \\
(0.069)\end{array}$ & $\begin{array}{c}0.748 \\
(0.066)\end{array}$ & $\begin{array}{c}0.395 \\
(0.217)\end{array}$ & $\begin{array}{c}0.402 \\
(0.088)\end{array}$ \\
\hline 1000 & $\begin{array}{c}5.923 \\
(0.735)\end{array}$ & $\begin{array}{c}1.915 \\
(0.377)\end{array}$ & $\begin{array}{c}0.799 \\
(0.046)\end{array}$ & $\begin{array}{c}0.762 \\
(0.045)\end{array}$ & $\begin{array}{c}0.384 \\
(0.146)\end{array}$ & $\begin{array}{c}0.406 \\
(0.065)\end{array}$ \\
\hline 3000 & $\begin{array}{c}5.963 \\
(0.429)\end{array}$ & $\begin{array}{c}1.976 \\
(0.218)\end{array}$ & $\begin{array}{c}0.808 \\
(0.026)\end{array}$ & $\begin{array}{c}0.772 \\
(0.025)\end{array}$ & $\begin{array}{c}0.390 \\
(0.083)\end{array}$ & $\begin{array}{c}0.416 \\
(0.037)\end{array}$ \\
\hline 5000 & $\begin{array}{c}5.979 \\
(0.336)\end{array}$ & $\begin{array}{c}1.988 \\
(0.172)\end{array}$ & $\begin{array}{c}0.810 \\
(0.021)\end{array}$ & $\begin{array}{c}0.775 \\
(0.021)\end{array}$ & $\begin{array}{c}0.389 \\
(0.064)\end{array}$ & $\begin{array}{c}0.418 \\
(0.029)\end{array}$ \\
\hline
\end{tabular}


Table 5.2: Realizations of estimators of parameters $\phi_{1}, \phi_{2}$, and $\phi_{3}$ and their standard deviations

\begin{tabular}{|c|c|c|c|c|c|c|}
\hline$\mu=0.5$ & $\nu=0.2$ & $\alpha=0.3$ & $\beta=0.1$ & $\phi_{1}=0.2$ & $\phi_{2}=0.2$ & $\phi_{3}=0.6$ \\
\hline$N$ & $\hat{\phi}_{1}^{c l s}$ & $\hat{\phi}_{1}^{Y W}$ & $\bar{\phi}_{2}^{c l s}$ & $\hat{\phi}_{2}^{Y W}$ & $\hat{\phi}_{3}^{c l s}$ & $\hat{\phi}_{3}^{Y W}$ \\
\hline 500 & $\begin{array}{c}0.177 \\
(0.287)\end{array}$ & $\begin{array}{c}0.193 \\
(0.332)\end{array}$ & $\begin{array}{c}0.175 \\
(0.482)\end{array}$ & $\begin{array}{c}0.186 \\
(0.494)\end{array}$ & $\begin{array}{c}0.648 \\
(0.591)\end{array}$ & $\begin{array}{c}0.621 \\
(0.490)\end{array}$ \\
\hline 1000 & $\begin{array}{c}0.196 \\
(0.141)\end{array}$ & $\begin{array}{c}0.195 \\
(0.125)\end{array}$ & $\begin{array}{c}0.181 \\
(0.140)\end{array}$ & $\begin{array}{c}0.184 \\
(0.125)\end{array}$ & $\begin{array}{c}0.622 \\
(0.157)\end{array}$ & $\begin{array}{c}0.621 \\
(0.141)\end{array}$ \\
\hline 3000 & $\begin{array}{c}0.196 \\
(0.075)\end{array}$ & $\begin{array}{c}0.197 \\
(0.069)\end{array}$ & $\begin{array}{c}0.196 \\
(0.077)\end{array}$ & $\begin{array}{c}0.196 \\
(0.071)\end{array}$ & $\begin{array}{c}0.608 \\
(0.088)\end{array}$ & $\begin{array}{c}0.607 \\
(0.081)\end{array}$ \\
\hline 5000 & $\begin{array}{c}0.198 \\
(0.056)\end{array}$ & $\begin{array}{c}0.199 \\
(0.052)\end{array}$ & $\begin{array}{c}0.199 \\
(0.057)\end{array}$ & $\begin{array}{c}0.199 \\
(0.053)\end{array}$ & $\begin{array}{c}0.603 \\
(0.065)\end{array}$ & $\begin{array}{c}0.602 \\
(0.060)\end{array}$ \\
\hline$\mu=1$ & $\nu=2$ & $\alpha=0.3$ & $\beta=0.5$ & $\phi_{1}=0.1$ & $\phi_{2}=0.7$ & $\phi_{3}=0.2$ \\
\hline$N$ & $\hat{\phi}_{1}^{c l s}$ & $\hat{\phi}_{1}^{Y W}$ & $\hat{\phi}_{2}^{c l s}$ & $\hat{\phi}_{2}^{Y W}$ & $\hat{\phi}_{3}^{c l s}$ & $\hat{\phi}_{3}^{Y W}$ \\
\hline 500 & $\begin{array}{c}0.233 \\
(0.652)\end{array}$ & $\begin{array}{c}0.091 \\
(0.282)\end{array}$ & $\begin{array}{c}0.628 \\
(0.645)\end{array}$ & $\begin{array}{c}0.723 \\
(0.335)\end{array}$ & $\begin{array}{c}0.140 \\
(0.756)\end{array}$ & $\begin{array}{c}0.187 \\
(0.292)\end{array}$ \\
\hline 1000 & $\begin{array}{c}0.352 \\
(0.350)\end{array}$ & $\begin{array}{c}0.103 \\
(0.083)\end{array}$ & $\begin{array}{c}0.214 \\
(0.223)\end{array}$ & $\begin{array}{c}0.699 \\
(0.096)\end{array}$ & $\begin{array}{c}0.435 \\
(0.119)\end{array}$ & $\begin{array}{c}0.198 \\
(0.081)\end{array}$ \\
\hline 3000 & $\begin{array}{c}0.089 \\
(0.089)\end{array}$ & $\begin{array}{c}0.107 \\
(0.046)\end{array}$ & $\begin{array}{c}0.719 \\
(0.101)\end{array}$ & $\begin{array}{c}0.692 \\
(0.051)\end{array}$ & $\begin{array}{c}0.192 \\
(0.084)\end{array}$ & $\begin{array}{c}0.201 \\
(0.046)\end{array}$ \\
\hline 5000 & $\begin{array}{c}0.092 \\
(0.061)\end{array}$ & $\begin{array}{c}0.106 \\
(0.035)\end{array}$ & $\begin{array}{c}0.711 \\
(0.068)\end{array}$ & $\begin{array}{c}0.691 \\
(0.038)\end{array}$ & $\begin{array}{c}0.197 \\
(0.058)\end{array}$ & $\begin{array}{c}0.203 \\
(0.034)\end{array}$ \\
\hline$\mu=4$ & $\nu=10$ & $\alpha=0.7$ & $\beta=0.9$ & $\phi_{1}=0.3$ & $\phi_{2}=0.4$ & $\phi_{3}=0.3$ \\
\hline $\bar{N}$ & $\hat{\phi}_{1}^{c l s}$ & $\bar{\phi}_{1}^{Y W}$ & $\hat{\phi}_{2}^{c l s}$ & $\hat{\phi}_{2}^{Y W}$ & $\hat{\phi}_{3}^{c l s}$ & $\hat{\phi}_{3}^{Y W}$ \\
\hline 500 & $\begin{array}{c}0.304 \\
(0.223)\end{array}$ & $\begin{array}{c}0.300 \\
(0.079)\end{array}$ & $\begin{array}{c}0.422 \\
(0.345)\end{array}$ & $\begin{array}{c}0.398 \\
(0.078)\end{array}$ & $\begin{array}{c}0.274 \\
(0.298)\end{array}$ & $\begin{array}{c}0.301 \\
(0.077)\end{array}$ \\
\hline 1000 & $\begin{array}{c}0.303 \\
(0.068)\end{array}$ & $\begin{array}{c}0.301 \\
(0.055)\end{array}$ & $\begin{array}{c}0.401 \\
(0.068)\end{array}$ & $\begin{array}{c}0.397 \\
(0.055)\end{array}$ & $\begin{array}{c}0.296 \\
(0.070)\end{array}$ & $\begin{array}{c}0.302 \\
(0.055)\end{array}$ \\
\hline 3000 & $\begin{array}{c}0.302 \\
(0.039)\end{array}$ & $\begin{array}{c}0.301 \\
(0.033)\end{array}$ & $\begin{array}{c}0.400 \\
(0.040)\end{array}$ & $\begin{array}{c}0.398 \\
(0.034)\end{array}$ & $\begin{array}{c}0.298 \\
(0.038)\end{array}$ & $\begin{array}{c}0.302 \\
(0.032)\end{array}$ \\
\hline 5000 & $\begin{array}{c}0.301 \\
(0.029)\end{array}$ & $\begin{array}{c}0.300 \\
(0.024)\end{array}$ & $\begin{array}{c}0.400 \\
(0.030)\end{array}$ & $\begin{array}{c}0.397 \\
(0.026)\end{array}$ & $\begin{array}{c}0.299 \\
(0.030)\end{array}$ & $\begin{array}{c}0.302 \\
(0.025)\end{array}$ \\
\hline$\mu=6$ & $\nu=2$ & $\alpha=0.8$ & $\beta=0.4$ & $\phi_{1}=0.5$ & $\phi_{2}=0.33$ & $\phi_{3}=0.17$ \\
\hline$N$ & $\hat{\phi}_{1}^{c l s}$ & $\hat{\phi}_{1}^{Y W}$ & $\hat{\phi}_{2}^{c l s}$ & $\hat{\phi}_{2}^{Y W}$ & $\hat{\phi}_{3}^{c l s}$ & $\hat{\phi}_{3}^{Y W}$ \\
\hline 500 & $\begin{array}{c}0.437 \\
(0.097)\end{array}$ & $\begin{array}{c}0.498 \\
(0.094)\end{array}$ & $\begin{array}{c}0.387 \\
(0.095)\end{array}$ & $\begin{array}{c}0.326 \\
(0.093)\end{array}$ & $\begin{array}{c}0.176 \\
(0.085)\end{array}$ & $\begin{array}{c}0.176 \\
(0.083)\end{array}$ \\
\hline 1000 & $\begin{array}{c}0.552 \\
(0.071)\end{array}$ & $\begin{array}{c}0.496 \\
(0.069)\end{array}$ & $\begin{array}{c}0.356 \\
(0.071)\end{array}$ & $\begin{array}{c}0.329 \\
(0.069)\end{array}$ & $\begin{array}{c}0.091 \\
(0.063)\end{array}$ & $\begin{array}{c}0.175 \\
(0.061)\end{array}$ \\
\hline 3000 & $\begin{array}{c}0.511 \\
(0.040)\end{array}$ & $\begin{array}{c}0.493 \\
(0.039)\end{array}$ & $\begin{array}{c}0.329 \\
(0.042)\end{array}$ & $\begin{array}{c}0.330 \\
(0.041)\end{array}$ & $\begin{array}{c}0.160 \\
(0.037)\end{array}$ & $\begin{array}{c}0.177 \\
(0.035)\end{array}$ \\
\hline 5000 & $\begin{array}{c}0.505 \\
(0.032)\end{array}$ & $\begin{array}{c}0.492 \\
(0.031)\end{array}$ & $\begin{array}{c}0.332 \\
(0.033)\end{array}$ & $\begin{array}{c}0.331 \\
(0.032)\end{array}$ & $\begin{array}{c}0.163 \\
(0.030)\end{array}$ & $\begin{array}{c}0.177 \\
(0.029)\end{array}$ \\
\hline
\end{tabular}




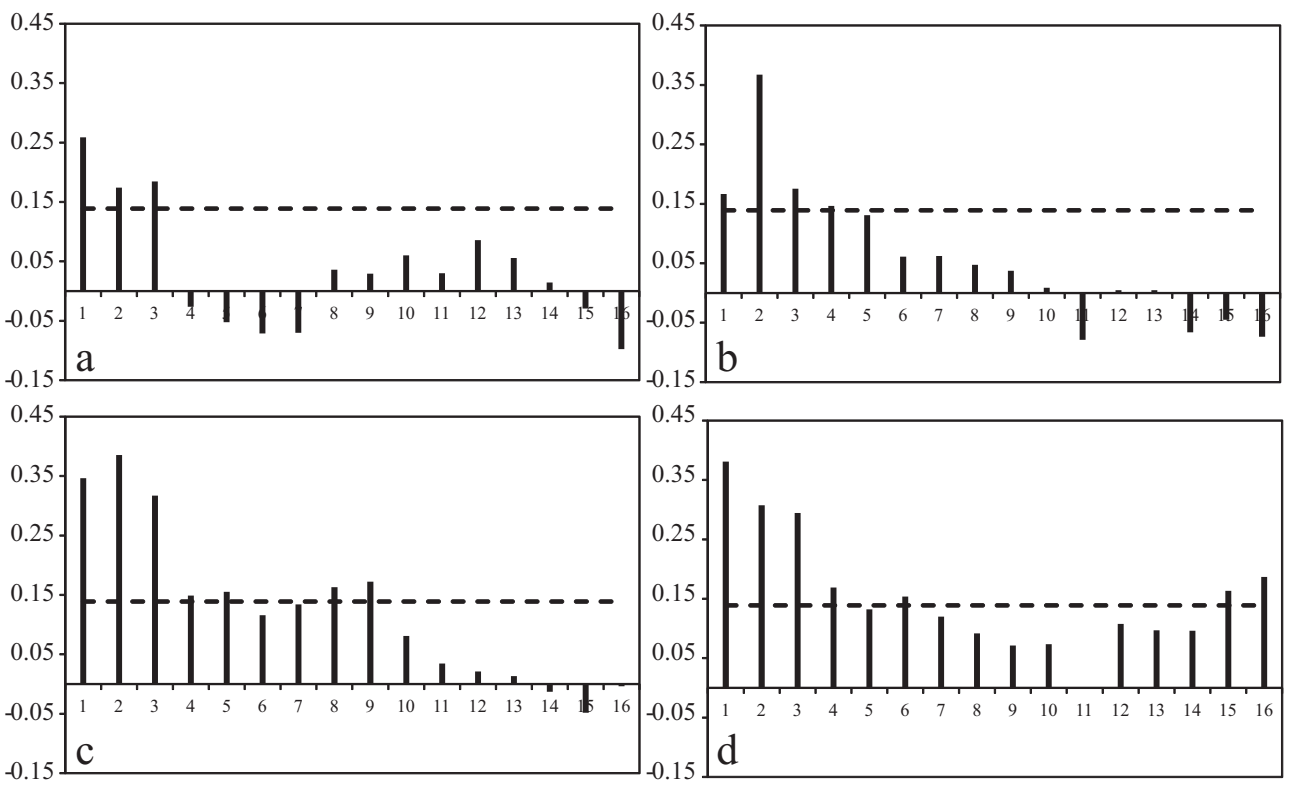

Fig. 5.2: Auto-correlation functions of simulated time series with $\mu=0.5, \nu=0.2$, $\alpha=0.3, \beta=0.1$ (a), $\mu=1, \nu=2, \alpha=0.3, \beta=0.5$ (b), $\mu=4, \nu=10, \alpha=0.7$, $\beta=0.9$ (c) and $\mu=8, \nu=2, \alpha=0.8, \beta=0.4$ (d)

method are a bit more precise, since their values are closer to the true values, but, on the other hand, estimators obtained by a Yule-Walker method are a bit more stabile, due to a smaller standard deviation. In the case of the estimation of parameters $\phi_{i}, i=1,2,3$, we could say that the methods are equally successful, regarding both, precision and stability.

Also, Figures 5.3 and 5.4 illustrate tendency of all estimators toward normal distribution.

\subsection{Real-data application}

Time series based on thinning have found their application in many fields of real life thanks to the ability to model a number of random events or elements of a population whose changes could be caused by surviving of existing elements, new incomers or new elements that could be results of interactions of existing ones. Originally obtained as a difference of two INAR series, CSDLINAR model could be adequate for comparing two systems or two phenomena with the mentioned properties. Here, we focused on the analysis of criminal data. We would like to compare the efficacy of two police stations in handling different kind of offenses. The data represents the difference in number of offences reported monthly to two police stations in Rochester, New York, USA, starting January 1991 to December 2001. The data are available 

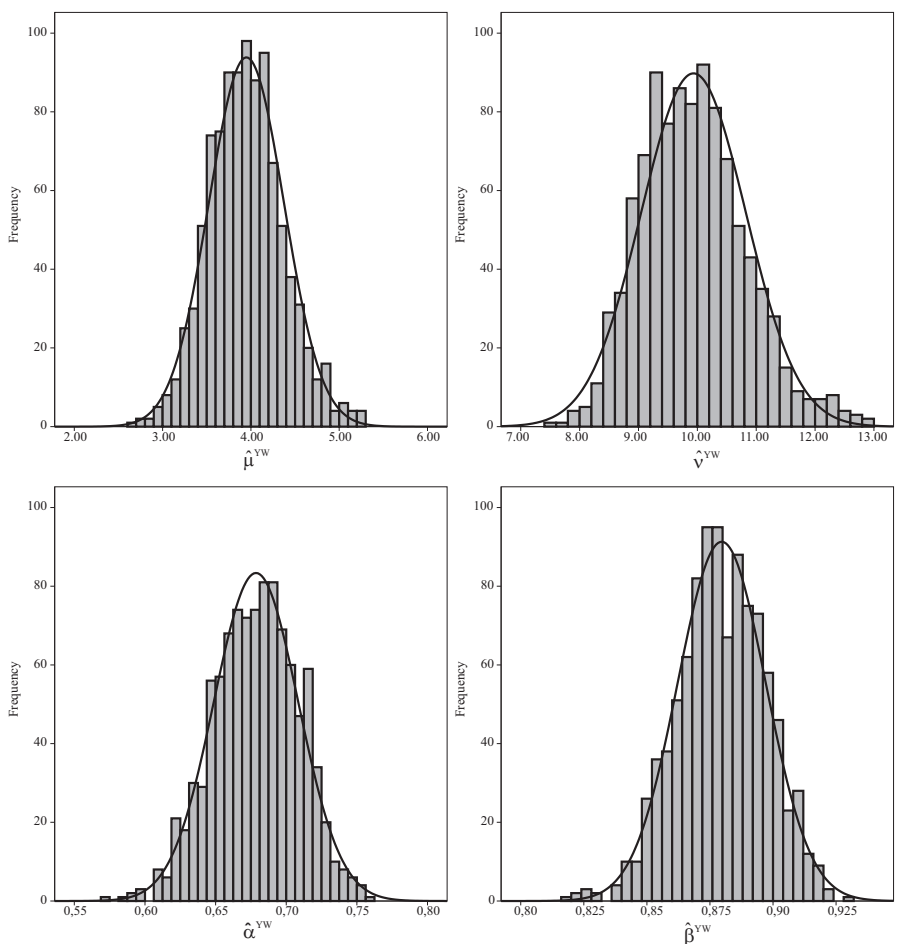

FIG. 5.3: Distribution of realizations of Yule-Walker estimators of parameters $\mu=4$, $\nu=10, \alpha=0.7$ and $\beta=0.9$
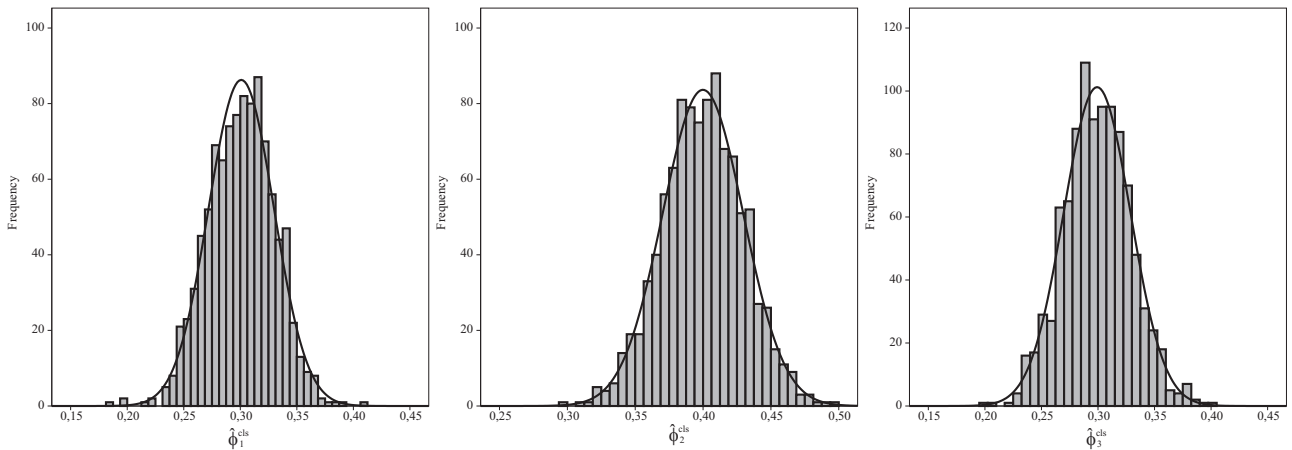

FIG. 5.4: Distribution of realizations of condition least squares estimators of parameters $\phi_{1}=0.3, \phi_{2}=0.4$ and $\phi_{3}=0.3$ 
on-line at The Forecasting Principles site (http://www.forecastingprinciples.com), in the Crime data section. First we considered the number of drug offences re-
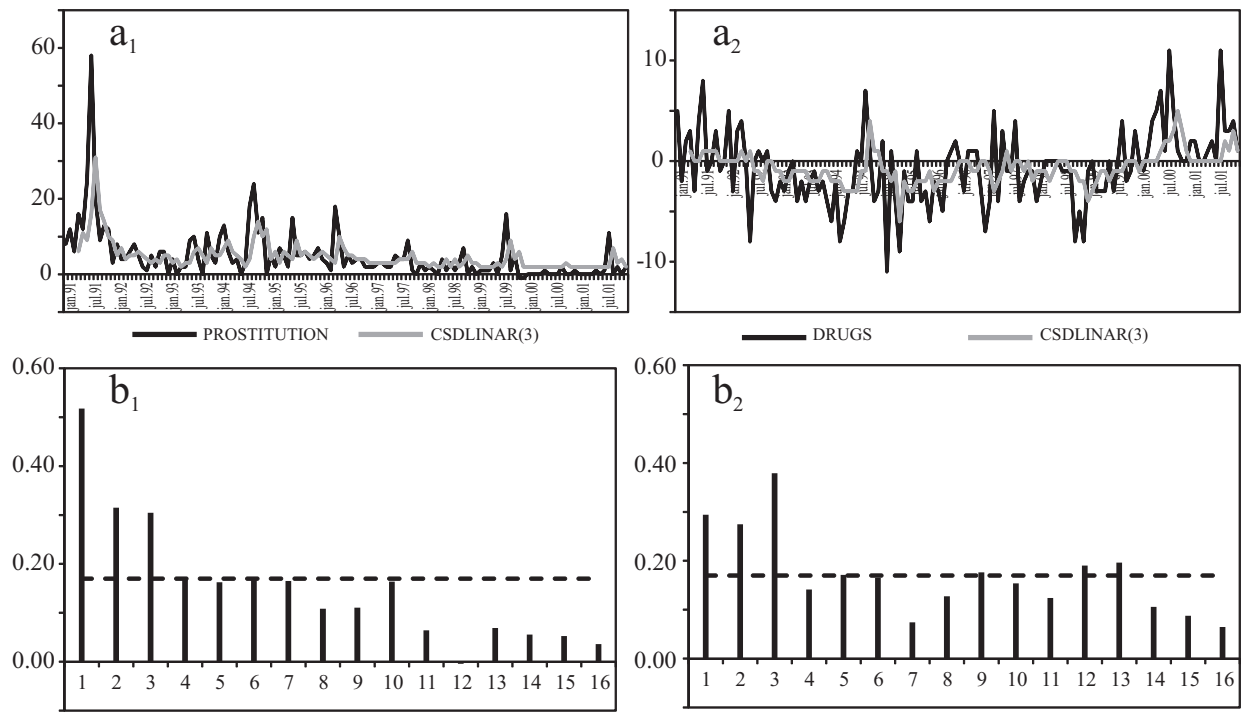

FIG. 5.5: Differences in numbers of reported prostitution incidents between police stations 36055001600 and 36055006000 (a1) and drug offences between police stations 36055001600 and 36055009500 (a2) and auto-correlation functions (a2, b2), respectively

ported to the police stations 36055001600 and 36055009500 and then, we considered the incidence of prostitution reported to the police stations 36055001600 and 36055006000 . In order to show advantages and defects of the CSDLINAR(p) model, we compared it with some existing models, in this case with $C I N A R S(p)$ model, introduced in [25], with the SDLINAR model of order one, previously introduced in [8] and with CSDLINAR(2). Models of order one and two are presented to demonstrate how fitting performances are rising as the order of model is getting higher. In the table 5.3, parameter estimates and the RMSE goodness of fit criterion for each of chosen models were showed. Also, on the Figure 5.5, the trajectories of the data and the corresponding CSDLINAR(p) model and values of the auto-correlation functions of the realized samples were showed.

According to the RMSE criterion, we could say that, for chosen data, the CSDLINAR(3) model is more appropriate then other two models. It could be seen that CSDLINAR model follows well the pattern of considered data. But, also, it should be mentioned that, due to a similar form of the conditional expectation, the CINARS and CSDLINAR models differs slightly and that their predictions differ in very few points. 
Table 5.3: Estimated parameters and RMSE for the CINARS(3), SDLINAR(1), CSDLINAR(2) and CSDLINAR(3) models

\begin{tabular}{|c|c|c|c|c|}
\hline & \multicolumn{2}{|l|}{ Drugs } & \multicolumn{2}{|l|}{ Prostitution } \\
\hline Model & Parameter estimates & RMSE & Parameter estimates & RMSE \\
\hline$C I N A R S(3)$ & $\begin{array}{c}\hat{\alpha}_{1}=0.172 \\
\hat{\alpha}_{2}=0.140 \\
\hat{\alpha}_{3}=0.298 \\
\hat{\mu}_{\varepsilon}=-0.230\end{array}$ & 3.265 & $\begin{array}{c}\hat{\alpha}_{1}=0.476 \\
\hat{\alpha}_{2}=-0.016 \\
\hat{\alpha}_{3}=0.168 \\
\hat{\mu}_{\varepsilon}=1.829\end{array}$ & 5.822 \\
\hline$S D L I N A R(1)$ & $\begin{array}{l}\hat{\mu}=1.797 \\
\hat{\nu}=2.411 \\
\hat{\alpha}=0.643 \\
\hat{\beta}=0.638\end{array}$ & 3.647 & $\begin{array}{l}\hat{\mu}=6.209 \\
\hat{\nu}=1.293 \\
\hat{\alpha}=0.578 \\
\hat{\beta}=0.564\end{array}$ & 5.901 \\
\hline$C S D L I N A R(2)$ & $\begin{array}{l}\hat{\mu}=1.751 \\
\hat{\nu}=2.446 \\
\hat{\alpha}=0.349 \\
\hat{\beta}=0.376 \\
\hat{\phi}_{1}=0.502 \\
\hat{\phi}_{2}=0.498\end{array}$ & 3.320 & $\begin{array}{c}\hat{\mu}=6.173 \\
\hat{\nu}=1.431 \\
\hat{\alpha}=0.553 \\
\hat{\beta}=0.321 \\
\hat{\phi}_{1}=0.870 \\
\hat{\phi}_{2}=0.130\end{array}$ & 5.897 \\
\hline$C S D L I N A R(3)$ & $\begin{array}{l}\hat{\mu}=1.751 \\
\hat{\nu}=2.446 \\
\hat{\alpha}=0.502 \\
\hat{\beta}=0.522 \\
\hat{\phi}_{1}=0.280 \\
\hat{\phi}_{2}=0.252 \\
\hat{\phi}_{3}=0.468\end{array}$ & 3.255 & $\begin{array}{l}\hat{\mu}=6.173 \\
\hat{\nu}=1.431 \\
\hat{\alpha}=0.625 \\
\hat{\beta}=0.492 \\
\hat{\phi}_{1}=0.718 \\
\hat{\phi}_{2}=0.100 \\
\hat{\phi}_{3}=0.182\end{array}$ & 5.771 \\
\hline
\end{tabular}

\subsection{Identification and prediction of latent components}

The fact that that the auto-correlation functions of $C G I N A R$ processes $\left\{X_{n}\right\}$ and $\left\{Y_{n}\right\}$ can be expressed through the terms of $Z_{n}$ and the expression for expectation of random variables $X_{n+k}$ and $Y_{n+k}$ conditioned on the random variable $Z_{n}$, given in the proof of the Proposition 3.5, open the possibility to identify and reconstruct or, even, predict the latent components $\left\{X_{n}\right\}$ and $\left\{Y_{n}\right\}$ of the CSDLINAR process $\left\{Z_{n}\right\}$ only on the basis of realization of the time series $\left\{Z_{n}\right\}$. Namely, the expectations of random variables $X_{n+k}$ and $Y_{n+k}$ conditioned on the random variable $Z_{n}$ are

$$
\begin{aligned}
E\left(X_{n+k} \mid H_{n}\right) & =\left(1-\alpha^{k}\right) \mu+\alpha^{k} \frac{\mu \nu}{1+\mu+\nu}+\alpha^{k} \sum_{i=1}^{p} \phi_{i} Z_{n+1-i}^{+}, \\
E\left(Y_{n+k} \mid H_{n}\right) & =\left(1-\beta^{k}\right) \nu+\beta^{k} \frac{\mu \nu}{1+\mu+\nu}+\beta^{k} \sum_{i=1}^{p} \phi_{i} Z_{n+1-i}^{-} .
\end{aligned}
$$

For $k=0$, we can get the formula that allowed us to reconstruct the latent com- 

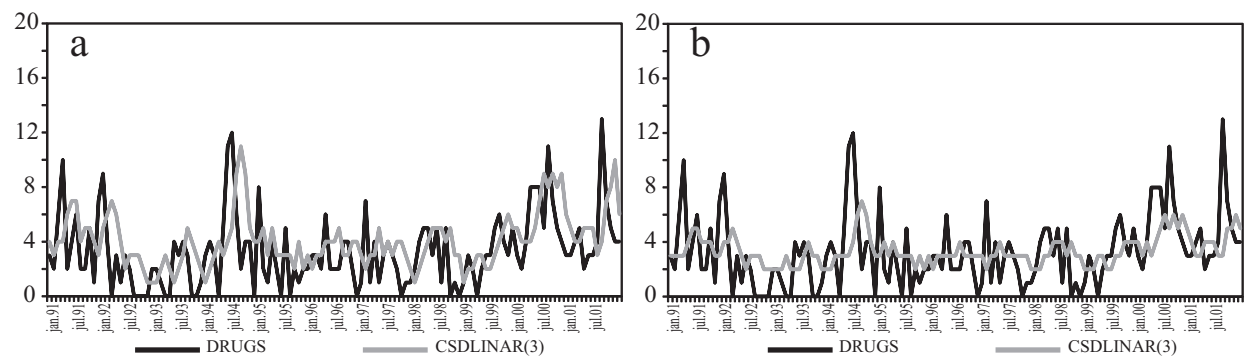

Fig. 5.6: Number of drug offenses reported to police station 36055001600 and reconstruction (a) and one step ahead prediction (b) models

ponents using only the realizations of time series $\left\{Z_{n}\right\}$

$$
\begin{aligned}
& \hat{X}_{n}=\hat{\alpha} \frac{\hat{\mu} \hat{\nu}}{1+\hat{\mu}+\hat{\nu}}+\sum_{i=1}^{p} \hat{\phi}_{i} Z_{n-i}^{+}, \\
& \hat{Y}_{n}=\hat{\beta} \frac{\hat{\mu} \hat{\nu}}{1+\hat{\mu}+\hat{\nu}}+\sum_{i=1}^{p} \hat{\phi}_{i} Z_{n-i}^{-} .
\end{aligned}
$$

For $k=1$, we get the formula that predicts the values of the latent components,

$$
\begin{aligned}
& \hat{X}_{n}=(1-\hat{\alpha}) \hat{\mu}+\hat{\alpha} \frac{\hat{\mu} \hat{\nu}}{1+\hat{\mu}+\hat{\nu}}+\hat{\alpha} \sum_{i=1}^{p} \hat{\phi}_{i} Z_{n-i}^{+}, \\
& \hat{Y}_{n}=(1-\hat{\beta}) \hat{\nu}+\hat{\beta} \frac{\hat{\mu} \hat{\nu}}{1+\hat{\mu}+\hat{\nu}}+\hat{\beta} \sum_{i=1}^{p} \hat{\phi}_{i} Z_{n-i}^{-} .
\end{aligned}
$$

In the case of our real data, the numbers of drug offences and prostitution incidents, we were able to check these formulas, since we already had the data from individual police stations. On the Figure 5.6, the number of drug offences reported to the police station 36055001600 and its reconstruction based on the realization of time series $\left\{Z_{n}\right\}$ (a) and its one step ahead prediction (b) are shown. The obtained RMSE values are 2.854 and 2.545 , respectively.

\section{Summary}

In this paper we have presented the construction and most important properties of the combined SDLINAR model of order $p$. Its marginal distribution is, in general, the skew discrete Laplace distribution. We have shown the Markovian property, strict stationarity and ergodicity of the presented model and, also, its conditional and correlation structure. The conditional least squares and Yule-Walker estimations have been derived and their strong consistency and asymptotic normality have been shown. In order to illustrate the asymptotic behavior, we have generated 1000 
samples of size 500, 1000, 3000 and 5000. On real-life data we have shown that this model has good fitting ability as well as the ability to reconstruct and predict latent components affecting it.

This model, by appropriate choice of its parameters, could be transformed to some other existing models. With $\phi_{1}=1$ it becomes the SDLINAR model of the first order presented in [8]. With $\alpha$ and $\beta$ that are equal, it transform to the STINAR model, proposed in [3]. If we equal $\alpha$ and $\beta$ and then $\mu$ and $\nu$ too, we get the symmetric DLINAR model introduced in [17]. With one of these parameters equal to zero, this model is reduced to the NGINAR model presented in [18].

A direction of following research could be increasing the dimension of the process and analyzing the model that represents s difference of two dependent NGINAR processes.

\section{R E F E R E N C E S}

1. M. A. Al-Osh, E. E. A. A. Aly: First order autoregressive time series with negative binomial and geometric marginals. Comm.Statist.Theory Methods. 21 (1992), 2483-2492.

2. M. A. Al-Osh, A. A. AlZAID: First-order integer-valued autoregressive (INAR(1)) process. J.Time Ser.Anal. 8 (1987), 261-275.

3. W. Barreto-Souza, M. Bourguignon: A skew INAR(1) process on Z. Advances in Statistical Analysis. 99 (2015), 189-208.

4. R. C. Bradley: Basic Properties of Strong Mixing Conditions. A Survey and Some Open Questions. Probability Surveys. 2 (2005), 107-144.

5. L. Breiman: Probability. The Society for Industrial and Applied Mathematics, Philadelphia, 1992.

6. P. J. Brockwell, R. A. Davis: Time Series - Theory and Methods. Springer, New York, 1991.

7. C. Chesneau, M. Kachour: A parametric study for the first-order signed integer-valued autoregressive process, Journal of Statistical Theory and Practice, 6(4) (2012), 760-782.

8. M. S. DJoRDJević: An extension on INAR models with discrete Laplace marginal distributions. Communication in Statistics - Theory and Methods. (in press)

9. Du Jin-GUAN, Li YUAN: The integer-valued autoregressive $(I N A R(p))$ model. J. Time Ser. Anal. 12 (1991), 129-142.

10. R. K. Freeland: True integer value time series. AStA Advances in Statistical Analysis. 94 (2010), 217-229.

11. R. K. Freeland, B. MCCABE: Asymptotic properties of CLS estimators in the Poisson AR(1) model. Statist.Probab.Lett. 73 (2005), 147-153.

12. M. Kachour, L. Truquet: A p-Order signed integer-valued autoregressive (SINAR(p)) model. Journal of Time Series Analysis. 32(3) (2011), 223-236.

13. H. Y. KIM, Y. PARK: A non-stationary integer-valued autoregressive model. Statist. Papers. 49 (2008), 485-502. 
14. E. McKenZIE: Some simple models for discrete variate time series. Water Resources Bulletin. 21 (1985), 645-650.

15. E. MCKEnZIE: Autoregressive moving-average processes with negative binomial and geometric distributions. Advances in Applied Probability. 18 (1986), 679705.

16. A. S. Nastić, M. M. Ristić, H. S. Bakouch: A combined geometric INAR $(p)$ model based on negative binomial thinning. Mathematical and Computer Modelling. 55 (2012), 1665-1672.

17. A. S. Nastić, M. M. Ristić, M. S. Djordjević: An INAR model with discrete Laplace marginal distributions. Brazilian Journal of Probability and Statistics. 30 (2016) 107-126.

18. M. M. Ristić, H. S. Bakouch, A. S. Nastić: A new geometric first-order integer-valued autoregressive (NGINAR(1)) process. J. Stat. Plan. Inference. 139 (2009), 2218-2226.

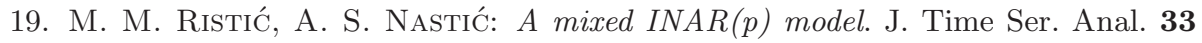
(2012), 903-915.

20. I. Silva, M. E. Silva: Asymptotic distribution of the Yule-Walker estimator for $\operatorname{INAR}(p)$ processes. Statistic and Probability Letters. 76(15), 1655-1663.

21. D. TృøSTheim: Estimation in nonlinear time series models. Stochastic Process Appl. 21 (1986), 251-273.

22. C. H. WeISS: The combined INAR( $p)$ models for time series of counts, Statist. Probab. Lett. 78 (2008), 1817-1822.

23. H. White: Asymptotic theory for econometrics. Academic Press, San Diego, 2001.

24. H. Zheng, I. V. BASAWA, S. DATTA: Inference for pth-order random coefficient integer-valued autoregressive processes. J. Time Ser. Anal. 27 (2006), 411-440.

25. H. ZhANG, D. WANG, F ZHU: Inference for INAR(p) processes with signed generalized power series thinning operator. Journal of Statistical Planning and Inference 140 (2010), 667-683

26. R. Zhu, H. JoE: Modelling count data time series with Markov processes based on binomial thinning. J. Time Series Anal. 27(5) (2006), 725-738.

\author{
Miodrag S. Djordjević \\ University of Niš \\ Faculty of Science and Mathematics \\ Department of Mathematics \\ Višegradska 33 \\ 18000 Niš, Serbia \\ dmiodrag@pmf.ni.ac.rs
}

